University of Nebraska - Lincoln

DigitalCommons@University of Nebraska - Lincoln

$2-22-2003$

\title{
Structures and stabilities of small silicon clusters: $A b$ initio molecular-orbital calculations of $\mathrm{Si}_{7}-\mathrm{Si}_{11}$
}

Xiaolei Zhu

University of Nebraska-Lincoln

Xiao Cheng Zeng

University of Nebraska-Lincoln, xzeng1@unl.edu

Follow this and additional works at: https://digitalcommons.unl.edu/chemzeng

Part of the Chemistry Commons

Zhu, Xiaolei and Zeng, Xiao Cheng, "Structures and stabilities of small silicon clusters: Ab initio molecularorbital calculations of $\mathrm{Si}_{7}-\mathrm{Si}_{11}$ " (2003). Xiao Cheng Zeng Publications. 36.

https://digitalcommons.unl.edu/chemzeng/36

This Article is brought to you for free and open access by the Published Research - Department of Chemistry at DigitalCommons@University of Nebraska - Lincoln. It has been accepted for inclusion in Xiao Cheng Zeng Publications by an authorized administrator of DigitalCommons@University of Nebraska - Lincoln. 


\title{
Structures and stabilities of small silicon clusters: $A b$ initio molecular-orbital calculations of $\mathrm{Si}_{7}-\mathrm{Si}_{11}$
}

\author{
Xiaolei Zhu and X. C. Zeng ${ }^{\text {a) }}$ \\ Department of Chemistry, University of Nebraska-Lincoln, Lincoln, Nebraska 68588
}

(Received 23 September 2002; accepted 14 November 2002)

\begin{abstract}
$A b$ initio all-electron molecular-orbital calculations have been carried out to study the structure and relative stability of small silicon clusters $\left(\mathrm{Si}_{n}, n=7-11\right)$. A number of low-energy geometric isomers are optimized at the second-order Møller-Plesset (MP2) MP2/6-31G(d) level. Harmonic vibrational analysis has been performed to assure that the optimized geometries are stable. The total energies of stable isomers are computed at the coupled-cluster single and double substitutions (including triple excitations) $[\mathrm{CCSD}(\mathrm{T})] \mathrm{CCSD}(\mathrm{T}) / 6-31 \mathrm{G}(d)$ level. The calculated binding energies per atom at both the MP2/6-31G(d) and $\operatorname{CCSD}(\mathrm{T}) / 6-31 \mathrm{G}(d)$ levels agree with the experiments. For $\mathrm{Si}_{7}, \mathrm{Si}_{8}$, and $\mathrm{Si}_{10}$, the lowest-energy structures are the same as those predicted previously from the all-electron optimization at the Hartree-Fock $(\mathrm{HF}) \mathrm{HF} / 6-31 \mathrm{G}(d)$ level [Raghavachari and Rohlfing, J. Chem. Phys. 89, 2219 (1988)]. For $\mathrm{Si}_{9}$, the lowest-energy isomer is same as that predicted based on density-functional plane-wave pseudopotential method [Vasiliev, Ogut, and Chelikowsky, Phys. Rev. Lett. 78, 4805 (1997)]. Particular attention has been given to $\mathrm{Si}_{11}$ because several low-energy geometric isomers were found nearly isoenergetic. On the basis of MP2/6-311G(2d)//CCSD(T)/6-311G(2d) calculation, we identified that the $C_{2 v}$ isomer, a tricapped trigonal prism with two additional caps on side trigonal faces, is most likely the global-minimum structure. However, another competitive geometric isomer for the global minimum is also found on basis of the MP2/6-311G(2d)//CCSD(T)/6-311G(2d) calculation. Additionally, calculations of the binding energy and the cluster polarizability offer more insights into relatively strong stability of two magic-number clusters $\mathrm{Si}_{6}$ and $\mathrm{Si}_{10}$. (c) 2003 American Institute of Physics.
\end{abstract}

[DOI: 10.1063/1.1535906]

\section{INTRODUCTION}

Since the advent of advanced laser vaporization techniques ${ }^{1-6}$ semiconductor atomic and nanoclusters have become an active subject of research, both experimentally and theoretically. ${ }^{1-50}$ A great deal of understanding has been obtained on this microscopic form of matter, for example, their selectivity, stability, and reactivity, and their evolution toward the bulk matter. Silicon atomic and nanoclusters have been extensively studied because of their relevance to the development of nanoelectronics. ${ }^{7-9}$ In particular, spectroscopic studies of atomic silicon clusters have been carried out by many groups. ${ }^{10-22}$ Photoelectron spectroscopy of silicon anion clusters ${ }^{10}$ indicates that silicon clusters with 4,6 , 7, and 10 atoms are closed-shell clusters. Photodissociation of silicon cation clusters ${ }^{11,12}$ demonstrates $\mathrm{Si}_{n}{ }^{+}$fragmentation products are mainly $\mathrm{Si}_{6}{ }^{+}-\mathrm{Si}_{11}{ }^{+}$. Several photodissociation $^{13-15}$ and collision-induced dissociation ${ }^{16-18}$ experiments have shown that $\mathrm{Si}_{6}$ and $\mathrm{Si}_{10}$ have exceptional stability, consistent with their "magic number" (particularly abundant) behavior in the mass spectra of Si clusters. ${ }^{19}$ The Raman $^{20}$ and infrared ${ }^{21}$ spectroscopy experiments have been utilized to infer the most stable structures of small silicon clusters. Mobility measurements ${ }^{22,23}$ have also provided evidences of various stable isomers. Note that all these spectroscopic measurements provide only indirect information on the structures of small silicon clusters. Nevertheless, spectro-

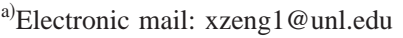

scopic measurements have motivated considerable theoretical and computational studies on the microscopic silicon clusters.

In principle, $a b$ initio molecular-orbital theory can provide direct structural information of the clusters. However, it is known that the number of stable geometric isomers of a cluster (local minima in the potential energy surface) increases exponentially with the number of atoms in the cluster. ${ }^{5}$ As such, determination of the globalminimum structure is a challenging problem. ${ }^{22,24}$ A series of systematic theoretical studies of small silicon clusters using all-electron molecular-orbital methods have been carried out by Raghavachari, Rohlfing, and their coworkers. ${ }^{21,25-30}$ Quantum Monte Carlo simulation, ${ }^{31}$ density-functional plane-wave pseudopotential methods, ${ }^{22,32-34,39-42}$ and other quantum mechanical means ${ }^{35-38,43-46}$ have also been employed by many groups to investigate various properties of silicon clusters and search for their global-minimum structures. Raghavachari and Logovinsky ${ }^{25}$ were apparently the first to use all-electron molecular-orbital methods to calculate energies of small silicon clusters $\left(\mathrm{Si}_{2}-\mathrm{Si}_{6}\right)$ and identify their lowest-energy geometries. Later, Raghavachari and Rohlfing ${ }^{27-30}$ studied larger silicon clusters, $\mathrm{Si}_{7}-\mathrm{Si}_{11}$. They performed geometry optimization of several low-energy geometric isomers of $\mathrm{Si}_{7}-\mathrm{Si}_{10}$ at the $\mathrm{HF} / 6-31 \mathrm{G}(d)$ level, followed by a total-energy calibration at the second-order Møller-Plesset (MP2), MP3, and MP4 levels with the polarized 6-31G $(d)$ basis set. ${ }^{27}$ The structures, bonding properties, and relative stability of $\mathrm{Si}_{7}-\mathrm{Si}_{10}$ clusters have been 
reported. In the cases of $\mathrm{Si}_{10}$ and $\mathrm{Si}_{11}$, more detailed study of several low-energy geometric isomers was undertaken at higher MP4 or quadratic configuration-interaction [QCISD(T) ] levels. ${ }^{28-30}$ For $\mathrm{Si}_{7}-\mathrm{Si}_{11}$, other theoretical methods have also been employed to search for their lowestenergy structures. ${ }^{21,26-31,35-45}$ Note that for $\mathrm{Si}_{11}$, there is still some uncertainty on the global-minimum structure due to sensitivity of low-energy structures and their energies to the selected theoretical methods (e.g., levels of theory and basis sets).

The aim of this work is to reexamine a number of lowenergy geometric isomers of $\mathrm{Si}_{7}-\mathrm{Si}_{11}$ that have been reported previously at the Hartree-Fock (HF) or densityfunctional levels of theory. We have used all-electron molecular-orbital methods with including electron correlation effects in the determination of the cluster geometry. The geometry optimization is at the MP2/6-31G $(d)$ level followed by the calibration of total energy at the $\operatorname{CCSD}(\mathrm{T}) / 6-31 \mathrm{G}(d)$ level. It is found that the electron correlation $^{47}$ can sometimes have qualitative effects on the cluster geometry as some previously obtained stable isomers at the HF level can became unstable at the MP2 level. This study also provides some additional insight into the relative stability of two magic-number clusters of silicon.

\section{COMPUTATIONAL DETAILS}

We used the Møller-Plesset perturbation to the second $\operatorname{order}^{51-53}$ with a polarized $6-31 \mathrm{G}(d)$ basis set, i.e., the MP2/6-31G $(d)$ level, to optimize the geometry of a number of low-energy isomers of $\mathrm{Si}_{7}-\mathrm{Si}_{11}$. Correlation effects of all electrons in the clusters are thus taken into account approximately. For the geometry optimization, the Berny algorithm $^{54}$ in the GAUSSIAN 98 software $^{55}$ is employed. This algorithm is designed to locate the local minimum but not the global minimum. Hence, a careful choice of initial isomeric structures of the cluster is important to seek possible candidate for the global-minimum isomer.

In this work, most geometric isomers of $\mathrm{Si}_{7}-\mathrm{Si}_{11}$ are taken from previously obtained by other groups on basis of either the HF level of theory or plane-wave pseudopotential techniques. To obtain the local-minimum structure unbiasly, first, a full geometry optimization without constraining the cluster symmetry is undertaken at the MP2/6-31G $(d)$ level. Second, the initial structures are optimized again with keeping the point-group symmetry of the clusters. Once the optimized structures are obtained from both approaches, the harmonic vibrational frequencies are then computed to examine possible appearance of any imaginary frequencies, that is, to examine whether the optimized structures are stable. Finally, for those stable isomers, their total energy is evaluated at the $\operatorname{CCSD}(\mathrm{T}) / 6-31 \mathrm{G}(d)$ level. $^{52,56-58}$

\section{RESULTS AND DISCUSSIONS}

Figure 1 displays the optimized geometries of several low-energy geometric isomers of $\mathrm{Si}_{7}-\mathrm{Si}_{11}$, where the silicon atoms are connected with a "bond" when the $\mathrm{Si}-\mathrm{Si}$ interatomic distance is less than $3.0 \AA$. The bond lengths are listed in Table I. In a benchmark calculation at the
MP2/6-31G $(d)$ level, the $\mathrm{Si}-\mathrm{Si}$ bond length of $\mathrm{Si}_{2}$ and $\mathrm{Si}_{2} \mathrm{H}_{6}$ are 2.26 and $2.34 \AA$, respectively, whereas the experimental values are 2.25 and $2.33 \AA^{25}$ This test calculation indicates that the relative percentage error of the calculated $\mathrm{Si}-\mathrm{Si}$ bond length is less than $0.5 \%$. Table II lists the calculated harmonic vibrational frequencies, IR intensities, and zero-point energy (ZPE). No imaginary frequencies were found for most of low-energy geometric isomers of $\mathrm{Si}_{7}-\mathrm{Si}_{11}$, at least at the MP2/6-31G(d) level of theory. Tables III and IV present the binding energies per atom at both the MP2/6-31G $(d)$ and $\operatorname{CCSD}(\mathrm{T}) / 6-31 \mathrm{G}(d)$ levels, together with the experimental values. As shown in Fig. 2, there is a good correlation between the calculated and the measured binding energies.

\section{A. Structure}

\section{1. $N=7$}

For $\mathrm{Si}_{7}$ we have examined a number of low-energy geometric isomers as those studied by Raghavachari and Rohlfing. ${ }^{27}$ No qualitative change in the geometry of these isomers is found except the capped trigonal prism, which no longer is a local minimum at the MP2/6-31G $(d)$ level. The lowest-energy isomer of $\mathrm{Si}_{7}$ is $7 a$, a pentagonal bipyrimid with $D_{5 h}$ symmetry, in agreement with the experiment ${ }^{20}$ and previous theoretical studies. ${ }^{27,28,35,36,39,42}$ Note that the pentagonal bipyrimid $\left(D_{5 h}\right)$ is also the global minimum structure of the argon cluster ${ }^{24}$ with seven atoms. Note also that the structures of small silicon clusters are different from the tetrahedral coordination characteristic of bulk silicon.

\section{2. $N=8$}

In the case of $\mathrm{Si}_{8}$, full geometry optimizations at the MP2/6-31G $(d)$ level followed by the total-energy calculation at the $\operatorname{CCSD}(\mathrm{T}) / 6-31 \mathrm{G}(d)$ level suggest that the isomer $8 a$, a distorted bicapped octahedron (capping two opposite faces) with $C_{2 h}\left({ }^{1} A_{g}\right)$ point group, is the lowest-energy structure. ${ }^{27,28,36,39,42}$ Raghavachari and Rohlfing ${ }^{27}$ have reported seven low-energy isomers of $\mathrm{Si}_{8}$ on the basis of the $\mathrm{HF} / 6-31 \mathrm{G}(d)$ level. Among the seven, six geometric isomers, i.e., $8 a\left(C_{2 h},{ }^{1} A_{g}\right), \quad 8 b\left(C_{2 v},{ }^{1} A_{1}\right), \quad 8 d\left(C_{2 v},{ }^{1} A_{1}\right)$, $8 e\left(D_{3 d},{ }^{3} A_{1 g}\right), 8 f\left(C_{3 v},{ }^{3} A_{2}\right)$, and $8 g\left(C_{s},{ }^{1} A^{\prime}\right)$ have the same structure as ours despite of some differences in energy ordering and geometric parameters due to different levels of theory. However, the bicapped-trigonal-prism $C_{2 v}$ isomer shown in Ref. 27 is no longer a local minimum due to the appearance of several imaginary frequencies at the MP2/6-31G $(d)$ level. Indeed, the full geometry optimization of the bicapped-trigonal-prism isomer yielded a new local-minimum structure ( $8 c$, with $C_{2}$ symmetry). The isomer $8 c$ could be viewed as a distorted $8 a$. Like the globalminimum isomer $8 a$, the isomer $8 h$ with $D_{2 d}$ symmetry is also a distorted bicapped octahedron but the caps are on adjacent faces. Our calculation indicates that $8 h$ is a relatively high- energy local minimum at the MP2/6-31G $(d)$ level. $8 h$ was previously thought to be the global minimum based on a semiempirical method. ${ }^{43}$ The isomer $8 g$, a singly- 


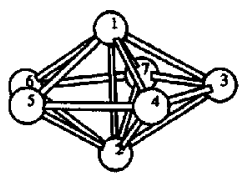

$7 a\left(D_{5 h}\right)$

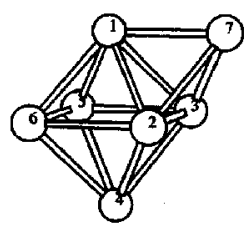

$7 c\left(C_{3 v}\right)$
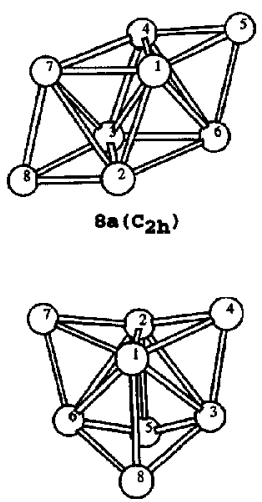

$8 b\left(c_{2 v}\right)$
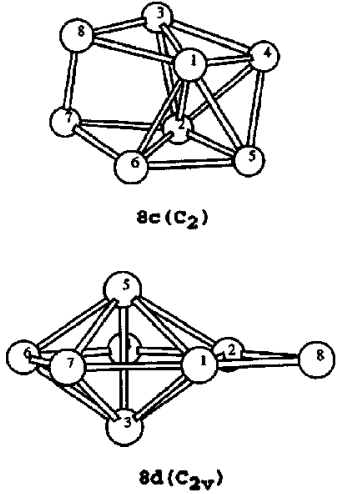

$8 d\left(C_{2 v}\right)$

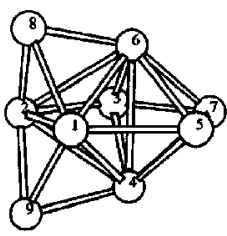

$9 \mathbf{a}\left(c_{2 \boldsymbol{v}}\right)$

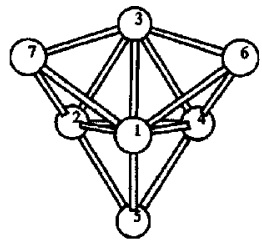

$7 b\left(c_{3 v}\right)$

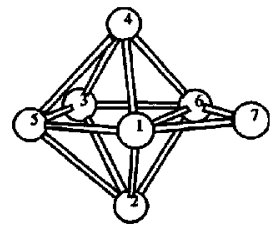

$7 a\left(c_{2 v}\right)$
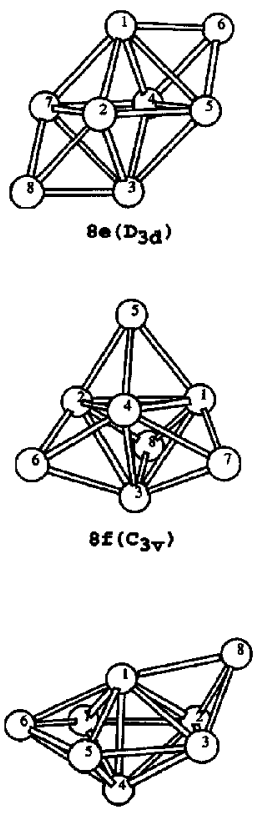

$8 g\left(C_{8}\right)$

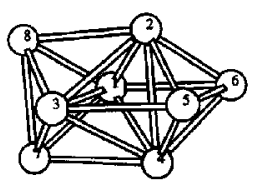

$\mathbf{8 h}\left(D_{2 d}\right)$

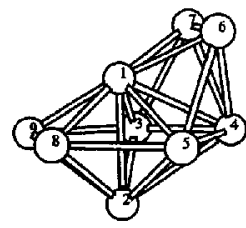

$9 b\left(c_{2 v}\right)$

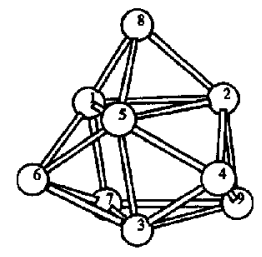

$\operatorname{se}\left(C_{s}\right)$

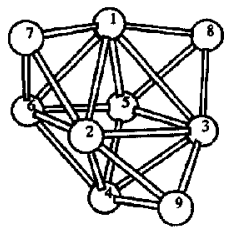

$9 d\left(C_{3 v}\right)$

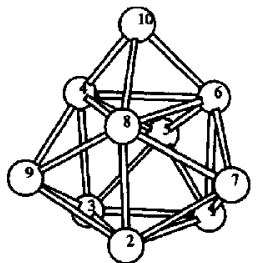

$10 a\left(c_{3 v}\right)$
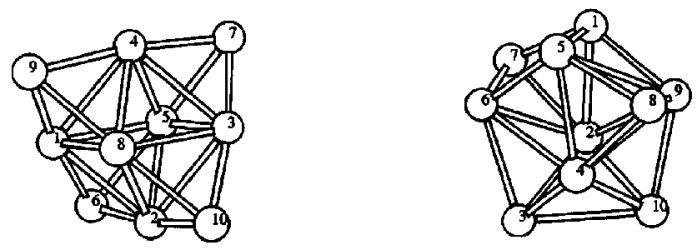

$10 b\left(T_{d}\right)$

$10 c\left(C_{2}\right)$

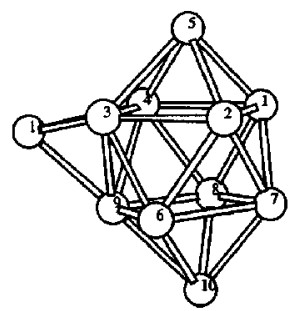

$\operatorname{11a}\left(c_{\mathrm{s}}\right)$

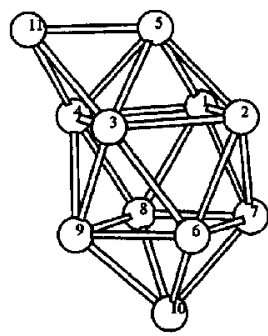

$11 e\left(C_{n}\right)$

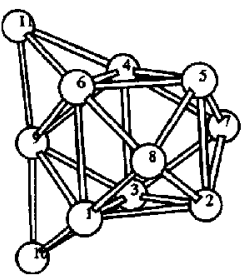

$11 b\left(c_{2 v}\right)$

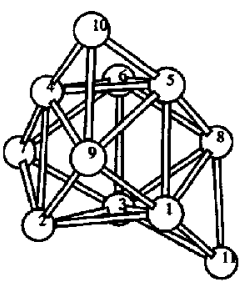

$11 d\left(C_{2}\right)$

FIG. 1. Geometries of the low-energy isomer structures of $\mathrm{Si}_{7}-\mathrm{Si}_{11}$ at the MP2/6-31G(d) level [11e at the MP2/6-311(2d) level].

capped pentagonal bipyramid, was also previously thought to be the global minimum based on the tight-binding molecular dynamics calculation. ${ }^{35}$
3. $N=9$

For $\mathrm{Si}_{9}$, the global-minimum isomer appears to be the $9 a\left(C_{2 v},{ }^{1} A_{1}\right)$, whose structure has been viewed as two 
TABLE I. Optimized geometry parameters for (a) low-energy geometric isomers of $\mathrm{Si}_{7}-\mathrm{Si}_{11}$ at the MP2/6-31G(d) level and (b) three low-energy isomers of $\mathrm{Si}_{11}$ at the MP2/6-311(2d) level.

\begin{tabular}{|c|c|c|c|c|c|c|c|c|c|}
\hline \multirow{2}{*}{$\begin{array}{l}\text { Cluster } \\
\text { (a) }\end{array}$} & \multirow[t]{2}{*}{ Isomer } & \multirow[t]{2}{*}{ PG (State) } & \multicolumn{2}{|c|}{ Bond length $(\AA)$} & \multirow[t]{2}{*}{ Cluster } & \multirow[t]{2}{*}{ Isomer } & \multirow[t]{2}{*}{ PG (State) } & \multicolumn{2}{|c|}{ Bond length $(\AA)$} \\
\hline & & & & & & & & $\mathrm{Si}_{2}-\mathrm{Si}_{8}$ & 2.373 \\
\hline \multirow[t]{21}{*}{$\mathrm{Si}_{7}$} & \multirow[t]{4}{*}{$7 a$} & \multirow[t]{4}{*}{$D_{5 h}\left({ }^{1} A_{1}^{\prime}\right)$} & & & & & & $\mathrm{Si}_{3}-\mathrm{Si}_{8}$ & 2.490 \\
\hline & & & $\mathrm{Si}_{1}-\mathrm{Si}_{4}$ & 2.458 & & $8 g$ & $C_{s}\left({ }^{1} A^{\prime}\right)$ & & \\
\hline & & & $\mathrm{Si}_{3}-\mathrm{Si}_{4}$ & 2.483 & & & & $\mathrm{Si}_{1}-\mathrm{Si}_{3}$ & 2.508 \\
\hline & & & $\mathrm{Si}_{1}-\mathrm{Si}_{2}$ & 2.512 & & & & $\mathrm{Si}_{1}-\mathrm{Si}_{7}$ & 2.524 \\
\hline & $7 b$ & $C_{3 v}\left({ }^{1} A_{1}\right)$ & & & & & & $\mathrm{Si}_{1}-\mathrm{Si}_{6}$ & 2.386 \\
\hline & & & $\mathrm{Si}_{1}-\mathrm{Si}_{4}$ & 2.485 & & & & $\mathrm{Si}_{1}-\mathrm{Si}_{4}$ & 2.478 \\
\hline & & & $\mathrm{Si}_{2}-\mathrm{Si}_{4}$ & 2.493 & & & & $\mathrm{Si}_{3}-\mathrm{Si}_{4}$ & 2.559 \\
\hline & & & $\mathrm{Si}_{1}-\mathrm{Si}_{7}$ & 2.558 & & & & $\mathrm{Si}_{4}-\mathrm{Si}_{7}$ & 2.422 \\
\hline & & & $\mathrm{Si}_{2}-\mathrm{Si}_{7}$ & 2.341 & & & & $\mathrm{Si}_{4}-\mathrm{Si}_{6}$ & 2.532 \\
\hline & $7 c$ & $C_{3 v}\left({ }^{1} A_{1}\right)$ & & & & & & $\mathrm{Si}_{2}-\mathrm{Si}_{3}$ & 2.897 \\
\hline & & & $\mathrm{Si}_{1}-\mathrm{Si}_{7}$ & 2.415 & & & & $\mathrm{Si}_{2}-\mathrm{Si}_{7}$ & 2.485 \\
\hline & & & $\mathrm{Si}_{1}-\mathrm{Si}_{2}$ & 2.452 & & & & $\mathrm{Si}_{6}-\mathrm{Si}_{7}$ & 2.440 \\
\hline & & & $\mathrm{Si}_{1}-\mathrm{Si}_{6}$ & 2.463 & & & & $\mathrm{Si}_{1}-\mathrm{Si}_{8}$ & 2.470 \\
\hline & & & $\mathrm{Si}_{4}-\mathrm{Si}_{6}$ & 2.555 & & & & $\mathrm{Si}_{3}-\mathrm{Si}_{8}$ & 2.403 \\
\hline & $7 d$ & $C_{2 v}\left({ }^{1} A_{1}\right)$ & & & & $8 h$ & $D_{2 d}\left({ }^{1} A_{1}\right)$ & & \\
\hline & & & $\mathrm{Si}_{1}-\mathrm{Si}_{7}$ & 2.293 & & & & $\mathrm{Si}_{1}-\mathrm{Si}_{7}$ & 2.343 \\
\hline & & & $\mathrm{Si}_{1}-\mathrm{Si}_{6}$ & 2.926 & & & & $\mathrm{Si}_{1}-\mathrm{Si}_{2}$ & 2.619 \\
\hline & & & $\mathrm{Si}_{1}-\mathrm{Si}_{5}$ & 2.415 & & & & $\mathrm{Si}_{4}-\mathrm{Si}_{7}$ & 2.926 \\
\hline & & & $\mathrm{Si}_{1}-\mathrm{Si}_{4}$ & 2.504 & & & & $\mathrm{Si}_{7}-\mathrm{Si}_{8}$ & 2.636 \\
\hline & & & $\mathrm{Si}_{3}-\mathrm{Si}_{5}$ & 2.315 & & & & $\mathrm{Si}_{2}-\mathrm{Si}_{4}$ & 2.894 \\
\hline & & & $\mathrm{Si}_{4}-\mathrm{Si}_{5}$ & 2.687 & $\mathrm{Si}_{9}$ & $9 a$ & $C_{2 v}\left({ }^{1} A_{1}\right)$ & & \\
\hline $\mathrm{Si}_{8}$ & $8 a$ & $C_{2 h}\left({ }^{1} A_{g}\right)$ & & & & & & $\mathrm{Si}_{5}-\mathrm{Si}_{7}$ & 2.401 \\
\hline & & & $\mathrm{Si}_{1}-\mathrm{Si}_{4}$ & 2.462 & & & & $\mathrm{Si}_{2}-\mathrm{Si}_{3}$ & 2.552 \\
\hline & & & $\mathrm{Si}_{4}-\mathrm{Si}_{6}$ & 2.865 & & & & $\mathrm{Si}_{1}-\mathrm{Si}_{2}$ & 2.547 \\
\hline & & & $\mathrm{Si}_{1}-\mathrm{Si}_{2}$ & 2.399 & & & & $\mathrm{Si}_{3}-\mathrm{Si}_{6}$ & 2.529 \\
\hline & & & $\mathrm{Si}_{2}-\mathrm{Si}_{6}$ & 2.750 & & & & $\mathrm{Si}_{6}-\mathrm{Si}_{7}$ & 2.391 \\
\hline & & & $\mathrm{Si}_{1}-\mathrm{Si}_{5}$ & 2.276 & & & & $\mathrm{Si}_{1}-\mathrm{Si}_{6}$ & 2.757 \\
\hline & & & $\mathrm{Si}_{5}-\mathrm{Si}_{6}$ & 2.454 & & & & $\mathrm{Si}_{2}-\mathrm{Si}_{8}$ & 2.345 \\
\hline & & & $\mathrm{Si}_{1}-\mathrm{Si}_{3}$ & 2.802 & & & & $\mathrm{Si}_{4}-\mathrm{Si}_{9}$ & 2.386 \\
\hline & $8 b$ & $C_{2 v}\left({ }^{1} A_{1}\right)$ & & & & & & $\mathrm{Si}_{4}-\mathrm{Si}_{6}$ & 2.976 \\
\hline & & & $\mathrm{Si}_{1}-\mathrm{Si}_{6}$ & 2.647 & & $9 b$ & $C_{2 v}\left({ }^{1} A_{1}\right)$ & & \\
\hline & & & $\mathrm{Si}_{1}-\mathrm{Si}_{2}$ & 2.480 & & & & $\mathrm{Si}_{1}-\mathrm{Si}_{4}$ & 2.644 \\
\hline & & & $\mathrm{Si}_{6}-\mathrm{Si}_{7}$ & 2.359 & & & & $\mathrm{Si}_{1}-\mathrm{Si}_{3}$ & 2.541 \\
\hline & & & $\mathrm{Si}_{1}-\mathrm{Si}_{7}$ & 2.384 & & & & $\mathrm{Si}_{1}-\mathrm{Si}_{7}$ & 2.370 \\
\hline & & & $\mathrm{Si}_{6}-\mathrm{Si}_{8}$ & 2.376 & & & & $\mathrm{Si}_{1}-\mathrm{Si}_{2}$ & 2.644 \\
\hline & & & $\mathrm{Si}_{1}-\mathrm{Si}_{8}$ & 2.656 & & & & $\mathrm{Si}_{2}-\mathrm{Si}_{4}$ & 2.751 \\
\hline & $8 c$ & $C_{2}\left({ }^{1} A\right)$ & & & & & & $\mathrm{Si}_{4}-\mathrm{Si}_{5}$ & 2.583 \\
\hline & & & $\mathrm{Si}_{4}-\mathrm{Si}_{5}$ & 2.536 & & & & $\mathrm{Si}_{4}-\mathrm{Si}_{6}$ & 2.525 \\
\hline & & & $\mathrm{Si}_{5}-\mathrm{Si}_{6}$ & 2.516 & & & & $\mathrm{Si}_{1}-\mathrm{Si}_{9}$ & 2.370 \\
\hline & & & $\mathrm{Si}_{2}-\mathrm{Si}_{4}$ & 2.503 & & & & $\mathrm{Si}_{8}-\mathrm{Si}_{9}$ & 2.404 \\
\hline & & & $\mathrm{Si}_{1}-\mathrm{Si}_{4}$ & 2.326 & & $9 c$ & $C_{s}\left({ }^{1} A^{\prime}\right)$ & & \\
\hline & & & $\mathrm{Si}_{2}-\mathrm{Si}_{3}$ & 2.487 & & & & $\mathrm{Si}_{3}-\mathrm{Si}_{7}$ & 2.458 \\
\hline & & & $\mathrm{Si}_{1}-\mathrm{Si}_{3}$ & 2.511 & & & & $\mathrm{Si}_{3}-\mathrm{Si}_{6}$ & 2.379 \\
\hline & & & $\mathrm{Si}_{1}-\mathrm{Si}_{2}$ & 2.649 & & & & $\mathrm{Si}_{6}-\mathrm{Si}_{7}$ & 2.656 \\
\hline & & & $\mathrm{Si}_{2}-\mathrm{Si}_{7}$ & 2.491 & & & & $\mathrm{Si}_{5}-\mathrm{Si}_{6}$ & 2.398 \\
\hline & & & $\mathrm{Si}_{3}-\mathrm{Si}_{8}$ & 2.483 & & & & $\mathrm{Si}_{1}-\mathrm{Si}_{6}$ & 2.441 \\
\hline & & & $\mathrm{Si}_{7}-\mathrm{Si}_{8}$ & 2.356 & & & & $\mathrm{Si}_{1}-\mathrm{Si}_{5}$ & 2.567 \\
\hline & $8 d$ & $C_{2 v}\left({ }^{1} A_{1}\right)$ & & & & & & $\mathrm{Si}_{1}-\mathrm{Si}_{8}$ & 2.376 \\
\hline & & & $\mathrm{Si}_{1}-\mathrm{Si}_{5}$ & 2.535 & & & & $\mathrm{Si}_{5}-\mathrm{Si}_{8}$ & 2.283 \\
\hline & & & $\mathrm{Si}_{4}-\mathrm{Si}_{5}$ & 2.482 & & & & $\mathrm{Si}_{7}-\mathrm{Si}_{9}$ & 2.972 \\
\hline & & & $\mathrm{Si}_{5}-\mathrm{Si}_{6}$ & 2.425 & & & & $\mathrm{Si}_{1}-\mathrm{Si}_{7}$ & 2.344 \\
\hline & & & $\mathrm{Si}_{3}-\mathrm{Si}_{5}$ & 2.695 & & & & $\mathrm{Si}_{3}-\mathrm{Si}_{5}$ & 2.464 \\
\hline & & & $\mathrm{Si}_{1}-\mathrm{Si}_{2}$ & 2.380 & & $9 d$ & $C_{3 v}\left({ }^{3} A_{2}\right)$ & & \\
\hline & & & $\mathrm{Si}_{2}-\mathrm{Si}_{4}$ & 2.483 & & & & $\mathrm{Si}_{1}-\mathrm{Si}_{5}$ & 2.493 \\
\hline & & & $\mathrm{Si}_{4}-\mathrm{Si}_{6}$ & 2.482 & & & & $\mathrm{Si}_{1}-\mathrm{Si}_{8}$ & 2.334 \\
\hline & & & $\mathrm{Si}_{1}-\mathrm{Si}_{8}$ & 2.320 & & & & $\mathrm{Si}_{1}-\mathrm{Si}_{3}$ & 2.953 \\
\hline & $8 e$ & $D_{3 d}\left({ }^{3} A_{1 g}\right)$ & & & & & & $\mathrm{Si}_{5}-\mathrm{Si}_{6}$ & 2.454 \\
\hline & & & $\mathrm{Si}_{1}-\mathrm{Si}_{4}$ & 2.574 & $\mathrm{Si}_{10}$ & $10 a$ & $C_{3 v}\left({ }^{1} A_{1}\right)$ & & \\
\hline & & & $\mathrm{Si}_{1}-\mathrm{Si}_{7}$ & 2.495 & & & & $\mathrm{Si}_{4}-\mathrm{Si}_{8}$ & 2.760 \\
\hline & & & $\mathrm{Si}_{1}-\mathrm{Si}_{6}$ & 2.368 & & & & $\mathrm{Si}_{3}-\mathrm{Si}_{4}$ & 2.491 \\
\hline & $8 f$ & $C_{3 v}\left({ }^{3} A_{2}\right)$ & & & & & & $\mathrm{Si}_{2}-\mathrm{Si}_{3}$ & 2.547 \\
\hline & & & $\mathrm{Si}_{2}-\mathrm{Si}_{4}$ & 2.674 & & & & $\mathrm{Si}_{4}-\mathrm{Si}_{10}$ & 2.318 \\
\hline & & & $\mathrm{Si}_{2}-\mathrm{Si}_{3}$ & 2.536 & & & & $\mathrm{Si}_{4}-\mathrm{Si}_{9}$ & 2.460 \\
\hline & & & $\mathrm{Si}_{2}-\mathrm{Si}_{5}$ & 2.469 & & & & $\mathrm{Si}_{3}-\mathrm{Si}_{9}$ & 2.434 \\
\hline
\end{tabular}


TABLE I. (Continued.)

\begin{tabular}{|c|c|c|c|c|c|c|c|c|c|}
\hline \multirow[t]{3}{*}{ Cluster } & \multirow{2}{*}{$\begin{array}{l}\text { Isomer } \\
10 b\end{array}$} & \multirow{2}{*}{$\begin{array}{c}\text { PG (State) } \\
T_{d}\left({ }^{1} A_{1}\right)\end{array}$} & \multicolumn{2}{|c|}{ Bond length $(\AA)$} & \multirow[t]{2}{*}{ Cluster } & \multirow[t]{2}{*}{ Isomer } & \multirow[t]{2}{*}{ PG (State) } & \multicolumn{2}{|c|}{ Bond length $(\AA)$} \\
\hline & & & & & & & & $\mathrm{Si}_{9}-\mathrm{Si}_{10}$ & 2.674 \\
\hline & & & $\mathrm{Si}_{4}-\mathrm{Si}_{5}$ & 2.581 & & $11 d$ & $C_{2}\left({ }^{1} A\right)$ & & \\
\hline & & & $\mathrm{Si}_{4}-\mathrm{Si}_{8}$ & 2.354 & & & & $\mathrm{Si}_{1}-\mathrm{Si}_{2}$ & 2.558 \\
\hline & $10 c$ & $C_{2}\left({ }^{1} A\right)$ & & & & & & $\mathrm{Si}_{1}-\mathrm{Si}_{3}$ & 2.441 \\
\hline & & & $\mathrm{Si}_{4}-\mathrm{Si}_{6}$ & 2.722 & & & & $\mathrm{Si}_{2}-\mathrm{Si}_{3}$ & 2.456 \\
\hline & & & $\mathrm{Si}_{4}-\mathrm{Si}_{9}$ & 2.708 & & & & $\mathrm{Si}_{1}-\mathrm{Si}_{5}$ & 2.601 \\
\hline & & & $\mathrm{Si}_{2}-\mathrm{Si}_{7}$ & 2.358 & & & & $\mathrm{Si}_{3}-\mathrm{Si}_{6}$ & 2.700 \\
\hline & & & $\mathrm{Si}_{1}-\mathrm{Si}_{2}$ & 2.508 & & & & $\mathrm{Si}_{3}-\mathrm{Si}_{7}$ & 2.385 \\
\hline & & & $\mathrm{Si}_{1}-\mathrm{Si}_{7}$ & 2.353 & & & & $\mathrm{Si}_{1}-\mathrm{Si}_{8}$ & 2.590 \\
\hline & & & $\mathrm{Si}_{5}-\mathrm{Si}_{6}$ & 2.420 & & & & $\mathrm{Si}_{3}-\mathrm{Si}_{8}$ & 2.459 \\
\hline & & & $\mathrm{Si}_{3}-\mathrm{Si}_{6}$ & 2.522 & & & & $\mathrm{Si}_{1}-\mathrm{Si}_{9}$ & 2.340 \\
\hline & & & $\mathrm{Si}_{3}-\mathrm{Si}_{10}$ & 2.695 & & & & $\mathrm{Si}_{4}-\mathrm{Si}_{10}$ & 2.428 \\
\hline & & & $\mathrm{Si}_{3}-\mathrm{Si}_{4}$ & 2.311 & & & & $\mathrm{Si}_{5}-\mathrm{Si}_{10}$ & 2.369 \\
\hline & & & $\mathrm{Si}_{4}-\mathrm{Si}_{10}$ & 2.473 & & & & $\mathrm{Si}_{9}-\mathrm{Si}_{10}$ & 2.504 \\
\hline & & & $\mathrm{Si}_{1}-\mathrm{Si}_{6}$ & 2.791 & & & & & \\
\hline & & & $\mathrm{Si}_{1}-\mathrm{Si}_{5}$ & 2.366 & (b) & & & & \\
\hline \multirow[t]{44}{*}{$\mathrm{Si}_{11}$} & $11 a$ & $C_{s}\left({ }^{1} A^{\prime}\right)$ & & & $\mathrm{Si}_{11}$ & $11 a^{\prime}$ & $C_{s}\left({ }^{1} A^{\prime}\right)$ & & \\
\hline & & & $\mathrm{Si}_{1}-\mathrm{Si}_{2}$ & 2.698 & & & & $\mathrm{Si}_{1}-\mathrm{Si}_{2}$ & 2.648 \\
\hline & & & $\mathrm{Si}_{2}-\mathrm{Si}_{3}$ & 2.531 & & & & $\mathrm{Si}_{2}-\mathrm{Si}_{3}$ & 2.504 \\
\hline & & & $\mathrm{Si}_{3}-\mathrm{Si}_{4}$ & 2.598 & & & & $\mathrm{Si}_{3}-\mathrm{Si}_{4}$ & 2.778 \\
\hline & & & $\mathrm{Si}_{1}-\mathrm{Si}_{5}$ & 2.450 & & & & $\mathrm{Si}_{1}-\mathrm{Si}_{5}$ & 2.468 \\
\hline & & & $\mathrm{Si}_{3}-\mathrm{Si}_{5}$ & 2.555 & & & & $\mathrm{Si}_{3}-\mathrm{Si}_{5}$ & 2.591 \\
\hline & & & $\mathrm{Si}_{3}-\mathrm{Si}_{6}$ & 2.412 & & & & $\mathrm{Si}_{3}-\mathrm{Si}_{6}$ & 2.405 \\
\hline & & & $\mathrm{Si}_{1}-\mathrm{Si}_{7}$ & 2.347 & & & & $\mathrm{Si}_{1}-\mathrm{Si}_{7}$ & 2.399 \\
\hline & & & $\mathrm{Si}_{1}-\mathrm{Si}_{8}$ & 2.419 & & & & $\mathrm{Si}_{1}-\mathrm{Si}_{8}$ & 2.442 \\
\hline & & & $\mathrm{Si}_{3}-\mathrm{Si}_{9}$ & 2.390 & & & & $\mathrm{Si}_{3}-\mathrm{Si}_{9}$ & 2.440 \\
\hline & & & $\mathrm{Si}_{3}-\mathrm{Si}_{11}$ & 2.483 & & & & $\mathrm{Si}_{3}-\mathrm{Si}_{11}$ & 2.493 \\
\hline & & & $\mathrm{Si}_{6}-\mathrm{Si}_{7}$ & 2.671 & & & & $\mathrm{Si}_{6}-\mathrm{Si}_{7}$ & 2.686 \\
\hline & & & $\mathrm{Si}_{6}-\mathrm{Si}_{9}$ & 2.481 & & & & $\mathrm{Si}_{6}-\mathrm{Si}_{9}$ & 2.519 \\
\hline & & & $\mathrm{Si}_{6}-\mathrm{Si}_{10}$ & 2.612 & & & & $\mathrm{Si}_{6}-\mathrm{Si}_{10}$ & 2.582 \\
\hline & & & $\mathrm{Si}_{7}-\mathrm{Si}_{10}$ & 2.369 & & & & $\mathrm{Si}_{7}-\mathrm{Si}_{10}$ & 2.416 \\
\hline & & & $\mathrm{Si}_{9}-\mathrm{Si}_{10}$ & 2.468 & & & & $\mathrm{Si}_{9}-\mathrm{Si}_{10}$ & 2.499 \\
\hline & & & $\mathrm{Si}_{9}-\mathrm{Si}_{11}$ & 2.365 & & & & $\mathrm{Si}_{9}-\mathrm{Si}_{11}$ & 2.391 \\
\hline & $11 b$ & $C_{2 v}\left({ }^{1} A_{1}\right)$ & & & & $11 b^{\prime}$ & $C_{2 v}\left({ }^{1} A_{1}\right)$ & & \\
\hline & & & $\mathrm{Si}_{2}-\mathrm{Si}_{7}$ & 2.501 & & & & $\mathrm{Si}_{2}-\mathrm{Si}_{7}$ & 2.511 \\
\hline & & & $\mathrm{Si}_{3}-\mathrm{Si}_{7}$ & 2.416 & & & & $\mathrm{Si}_{3}-\mathrm{Si}_{7}$ & 2.434 \\
\hline & & & $\mathrm{Si}_{2}-\mathrm{Si}_{5}$ & 2.566 & & & & $\mathrm{Si}_{2}-\mathrm{Si}_{5}$ & 2.594 \\
\hline & & & $\mathrm{Si}_{3}-\mathrm{Si}_{4}$ & 2.660 & & & & $\mathrm{Si}_{3}-\mathrm{Si}_{4}$ & 2.677 \\
\hline & & & $\mathrm{Si}_{1}-\mathrm{Si}_{3}$ & 2.479 & & & & $\mathrm{Si}_{1}-\mathrm{Si}_{3}$ & 2.524 \\
\hline & & & $\mathrm{Si}_{1}-\mathrm{Si}_{9}$ & 2.433 & & & & $\mathrm{Si}_{1}-\mathrm{Si}_{9}$ & 2.463 \\
\hline & & & $\mathrm{Si}_{1}-\mathrm{Si}_{10}$ & 2.414 & & & & $\mathrm{Si}_{1}-\mathrm{Si}_{10}$ & 2.442 \\
\hline & & & $\mathrm{Si}_{9}-\mathrm{Si}_{10}$ & 2.442 & & & & $\mathrm{Si}_{9}-\mathrm{Si}_{10}$ & 2.470 \\
\hline & & & $\mathrm{Si}_{1}-\mathrm{Si}_{2}$ & 2.475 & & & & $\mathrm{Si}_{1}-\mathrm{Si}_{2}$ & 2.506 \\
\hline & $11 c$ & $C_{s}\left({ }^{1} A^{\prime}\right)$ & & & & $11 e$ & $C_{s}\left({ }^{1} A^{\prime}\right)$ & & \\
\hline & & & $\mathrm{Si}_{2}-\mathrm{Si}_{3}$ & 2.690 & & & & $\mathrm{Si}_{7}-\mathrm{Si}_{10}$ & 2.459 \\
\hline & & & $\mathrm{Si}_{3}-\mathrm{Si}_{4}$ & 2.515 & & & & $\mathrm{Si}_{1}-\mathrm{Si}_{7}$ & 2.354 \\
\hline & & & $\mathrm{Si}_{1}-\mathrm{Si}_{5}$ & 2.369 & & & & $\mathrm{Si}_{1}-\mathrm{Si}_{2}$ & 2.603 \\
\hline & & & $\mathrm{Si}_{3}-\mathrm{Si}_{5}$ & 2.522 & & & & $\mathrm{Si}_{2}-\mathrm{Si}_{10}$ & 2.932 \\
\hline & & & $\mathrm{Si}_{2}-\mathrm{Si}_{6}$ & 2.333 & & & & $\mathrm{Si}_{1}-\mathrm{Si}_{7}$ & 2.502 \\
\hline & & & $\mathrm{Si}_{3}-\mathrm{Si}_{6}$ & 2.382 & & & & $\mathrm{Si}_{6}-\mathrm{Si}_{10}$ & 2.495 \\
\hline & & & $\mathrm{Si}_{1}-\mathrm{Si}_{7}$ & 2.372 & & & & $\mathrm{Si}_{1}-\mathrm{Si}_{5}$ & 2.429 \\
\hline & & & $\mathrm{Si}_{1}-\mathrm{Si}_{8}$ & 2.333 & & & & $\mathrm{Si}_{4}-\mathrm{Si}_{5}$ & 2.667 \\
\hline & & & $\mathrm{Si}_{4}-\mathrm{Si}_{8}$ & 2.382 & & & & $\mathrm{Si}_{3}-\mathrm{Si}_{6}$ & 2.458 \\
\hline & & & $\mathrm{Si}_{3}-\mathrm{Si}_{9}$ & 2.401 & & & & $\mathrm{Si}_{4}-\mathrm{Si}_{9}$ & 2.480 \\
\hline & & & $\mathrm{Si}_{3}-\mathrm{Si}_{11}$ & 2.455 & & & & $\mathrm{Si}_{4}-\mathrm{Si}_{11}$ & 2.451 \\
\hline & & & $\mathrm{Si}_{5}-\mathrm{Si}_{11}$ & 2.376 & & & & $\mathrm{Si}_{3}-\mathrm{Si}_{4}$ & 2.503 \\
\hline & & & $\mathrm{Si}_{6}-\mathrm{Si}_{7}$ & 2.673 & & & & $\mathrm{Si}_{1}-\mathrm{Si}_{4}$ & 2.576 \\
\hline & & & $\mathrm{Si}_{6}-\mathrm{Si}_{9}$ & 2.661 & & & & $\mathrm{Si}_{9}-\mathrm{Si}_{10}$ & 2.558 \\
\hline & & & $\mathrm{Si}_{6}-\mathrm{Si}_{10}$ & 2.447 & & & & $\mathrm{Si}_{5}-\mathrm{Si}_{11}$ & 2.478 \\
\hline & & & $\mathrm{Si}_{7}-\mathrm{Si}_{10}$ & 2.480 & & & & $\mathrm{Si}_{6}-\mathrm{Si}_{9}$ & 2.467 \\
\hline
\end{tabular}

stacked distorted rhombi with an additional atom capped on top. ${ }^{22,45}$ It can be also viewed as a bicapped pentagonal bipyramid. This lowest-energy structure was predicted by Vasiliev, Ogut, and Chelikowsky ${ }^{42}$ and later by other groups. ${ }^{38,45}$ The isomer $9 b\left(C_{2 v},{ }^{1} A_{1}\right)$, also a bicapped pentagonal bipyramid (but the two caps are on the same side of the pyramid), is a local minimum. $9 b$ can be obtained via a full geometry optimization starting from the $\left(D_{3 h},{ }^{1} A_{1}\right)$ tri- 
TABLE II. Vibrational frequencies $\left(\mathrm{cm}^{-1}\right)$, IR intensities, and zero-point energies of low-energy geometric isomers of $\mathrm{Si}_{7}-\mathrm{Si}_{11}$.

\begin{tabular}{|c|c|c|c|c|c|c|}
\hline Cluster & Isomer & PG (state) & \multicolumn{3}{|c|}{ Vibrational frequency (IR intensities) } & $\mathrm{ZPE}(\mathrm{eV})$ \\
\hline \multirow[t]{16}{*}{$\mathrm{Si}_{7}$} & $7 a$ & $D_{5 h}\left({ }^{1} A_{1}^{\prime}\right)$ & & & & \\
\hline & & & $232.54(0.21)$ & $261.75(0.15)$ & $443.38(10.52)$ & 0.31 \\
\hline & $7 b$ & $C_{3 v}\left({ }^{1} A_{1}\right)$ & & & & \\
\hline & & & $131.68(0.03)$ & $186.23(0.02)$ & $242.02(3.25)$ & 0.28 \\
\hline & & & $300.72(2.84)$ & $332.40(0.23)$ & $364.05(0.05)$ & \\
\hline & & & $365.61(0.003)$ & $459.62(3.40)$ & $531.29(12.68)$ & \\
\hline & $7 c$ & $C_{3 v}\left({ }^{1} A_{1}\right)$ & & & & \\
\hline & & & $137.84(1.44)$ & $181.66(0.54)$ & $283.53(0.93)$ & 0.31 \\
\hline & & & $301.88(3.01)$ & $332.30(0.91)$ & $368.55(3.93)$ & \\
\hline & & & 407.06(10.69) & $426.94(3.91)$ & $491.86(25.05)$ & \\
\hline & $7 d$ & $C_{2 v}\left({ }^{1} A_{1}\right)$ & & & & \\
\hline & & & $38.97(0.02)$ & $100.69(0.33)$ & $109.02(1.13)$ & 0.26 \\
\hline & & & $189.99(0.24)$ & $247.14(1.88)$ & $253.24(0.26)$ & \\
\hline & & & & & $355.39(21.04)$ & \\
\hline & & & $329.29(2.76)$ & $335.69(8.44)$ & $376.51(0.14)$ & \\
\hline & & & 449.01(13.54) & $469.22(0.73)$ & $501.24(2.28)$ & \\
\hline \multirow[t]{43}{*}{$\mathrm{Si}_{8}$} & $8 a$ & $C_{2 h}\left({ }^{1} A_{g}\right)$ & & & & \\
\hline & & & 75.32(0.09) & $150.67(0.70)$ & $174.11(0.88)$ & 0.34 \\
\hline & & & $255.47(0.54)$ & $318.59(4.63)$ & $385.50(0.59)$ & \\
\hline & & & $317.05(2.07)$ & & & \\
\hline & & & $409.01(8.52)$ & $544.93(8.35)$ & & \\
\hline & $8 b$ & $C_{2 v}\left({ }^{1} A_{1}\right)$ & & & & \\
\hline & & & $132.26(4.49)$ & $179.77(3.97)$ & $214.29(0.19)$ & 0.35 \\
\hline & & & $222.47(5.60)$ & $254.85(2.00)$ & $261.10(1.42)$ & \\
\hline & & & $292.59(0.35)$ & $303.69(0.01)$ & $319.27(3.02)$ & \\
\hline & & & $370.52(0.33)$ & $407.71(7.94)$ & $428.76(0.04)$ & \\
\hline & & & $445.17(0.2)$ & $477.61(0.50)$ & 498.07(0.38) & \\
\hline & $8 c$ & $C_{2}\left({ }^{1} A\right)$ & & & & \\
\hline & & & $90.04(1.39)$ & $123.25(0.02)$ & $172.61(0.21)$ & 0.32 \\
\hline & & & $177.92(0.48)$ & $186.86(1.19)$ & $201.84(0.19)$ & \\
\hline & & & $210.49(1.15)$ & $289.20(0.05)$ & $301.71(0.61)$ & \\
\hline & & & $325.50(4.87)$ & $330.55(0.54)$ & $338.80(0.001)$ & \\
\hline & & & $372.49(5.19)$ & $376.38(0.03)$ & $424.67(10.23)$ & \\
\hline & & & $461.55(6.35)$ & $488.39(6.84)$ & & \\
\hline & $8 d$ & $C_{2 v}\left({ }^{1} A_{1}\right)$ & & & & \\
\hline & & & $92.60(0.35)$ & $145.75(0.001)$ & $162.03(0.97)$ & 0.33 \\
\hline & & & $162.42(0.08)$ & $194.11(2.27)$ & $260.71(1.40)$ & \\
\hline & & & $274.41(0.52)$ & $284.94(0.51)$ & $310.04(0.31)$ & \\
\hline & & & $340.26(4.13)$ & $362.84(1.39)$ & $385.43(7.05)$ & \\
\hline & & & $408.50(0.06)$ & $420.07(7.49)$ & $439.13(9.88)$ & \\
\hline & & & $524.54(24.89)$ & & & \\
\hline & $8 e$ & $D_{3 d}\left({ }^{3} A_{1 g}\right)$ & & & & \\
\hline & & & $135.79(0.53)$ & $308.90(8.10)$ & $323.85(0.02)$ & 0.63 \\
\hline & & & $412.11(4.70)$ & $482.84(3.58)$ & & \\
\hline & $8 f$ & $C_{3 v}\left({ }^{3} A_{2}\right)$ & & & & \\
\hline & & & $140.58(0.26)$ & $230.19(0.05)$ & $230.65(0.06)$ & 0.34 \\
\hline & & & $248.87(0.004)$ & $251.79(4.51)$ & $268.42(2.16)$ & \\
\hline & & & $322.38(1.66)$ & $345.73(0.55)$ & $386.96(4.96)$ & \\
\hline & & & $437.36(4.17)$ & $463.54(5.78)$ & & \\
\hline & $8 g$ & $C_{s}\left({ }^{1} A^{\prime}\right)$ & & & & \\
\hline & & & $127.07(1.19)$ & $128.32(1.15)$ & $170.27(1.80)$ & 0.34 \\
\hline & & & $185.39(0.44)$ & $221.53(0.04)$ & $221.75(0.47)$ & \\
\hline & & & $252.09(1.69)$ & $261.79(1.89)$ & $295.76(0.66)$ & \\
\hline & & & $316.11(0.46)$ & $329.26(0.02)$ & $334.85(1.05)$ & \\
\hline & & & $357.38(1.64)$ & $399.79(0.12)$ & $425.12(2.62)$ & \\
\hline & & & $426.16(1.58)$ & $463.86(0.05)$ & $482.58(4.86)$ & \\
\hline & $8 h$ & $D_{2 d}\left({ }^{1} A_{1}\right)$ & $145.56(1.59)$ & & & \\
\hline & & & $276.74(4.34)$ & $316.27(0.01)$ & $326.41(1.03)$ & 0.38 \\
\hline & & & $468.87(0.53)$ & $496.35(0.001)$ & $616.34(18.34)$ & \\
\hline \multirow[t]{10}{*}{$\mathrm{Si}_{9}$} & $9 a$ & $C_{2 v}\left({ }^{1} A_{1}\right)$ & & & & \\
\hline & & & $91.92(2.29)$ & $119.14(3.33)$ & $172.73(0.96)$ & 0.39 \\
\hline & & & $203.75(4.54)$ & $216.59(1.70)$ & $248.10(0.01)$ & \\
\hline & & & $270.59(1.37)$ & $280.11(0.07)$ & $299.67(3.97)$ & \\
\hline & & & $305.60(1.57)$ & $323.10(2.83)$ & $338.33(1.50)$ & \\
\hline & & & $356.02(1.35)$ & $423.99(1.25)$ & $430.69(0.002)$ & \\
\hline & & & $452.31(1.47)$ & $488.95(1.96)$ & $498.22(1.76)$ & \\
\hline & $9 b$ & $C_{2 v}\left({ }^{1} A_{1}\right)$ & & & & \\
\hline & & & $134.13(0.13)$ & $165.82(0.001)$ & $173.83(1.22)$ & 0.37 \\
\hline & & & $181.72(0.27)$ & $233.35(0.83)$ & $257.63(0.001)$ & \\
\hline
\end{tabular}


TABLE II. (Continued.)

\begin{tabular}{|c|c|c|c|c|c|c|}
\hline \multirow[t]{2}{*}{ Cluster } & \multirow[t]{2}{*}{ Isomer } & \multirow[t]{2}{*}{ PG (state) } & \multicolumn{3}{|c|}{ Vibrational frequency (IR intensities) } & \multirow[t]{2}{*}{$\mathrm{ZPE}(\mathrm{eV})$} \\
\hline & & & $265.31(2.49)$ & $267.56(0.87)$ & $296.65(0.85)$ & \\
\hline & \multirow{11}{*}{$9 c$} & & $308.31(1.44)$ & $317.82(1.48)$ & $331.62(0.09)$ & \\
\hline & & & $367.38(2.02)$ & $369.82(2.65)$ & $408.56(0.004)$ & \\
\hline & & & $440.17(6.94)$ & $552.78(1.39)$ & & \\
\hline & & $C_{s}\left({ }^{1} A^{\prime}\right)$ & & & & \multirow{8}{*}{0.38} \\
\hline & & & $70.55(1.38)$ & $107.13(0.22)$ & $162.90(0.15)$ & \\
\hline & & & $166.91(1.95)$ & $205.91(0.25)$ & $238.60(3.57)$ & \\
\hline & & & $252.71(5.60)$ & $263.56(1.22)$ & $287.39(1.92)$ & \\
\hline & & & $295.07(2.84)$ & 304.44(1.97) & $308.15(1.37)$ & \\
\hline & & & $347.10(0.70)$ & $354.96(0.28)$ & $369.99(1.36)$ & \\
\hline & & & $405.09(0.35)$ & $452.67(3.80)$ & $460.95(6.73)$ & \\
\hline & & & $487.42(0.13)$ & $514.49(0.16)$ & & \\
\hline & \multirow[t]{5}{*}{$9 d$} & $C_{3 v}\left({ }^{3} A_{2}\right)$ & & & & \multirow{5}{*}{0.45} \\
\hline & & & $100.30(6.84)$ & $113.54(2.35)$ & 179.23(9.29) & \\
\hline & & & $208.35(0.64)$ & $283.20(1.64)$ & $324.14(0.60)$ & \\
\hline & & & $341.40(0.06)$ & $350.19(3.98)$ & 397.04(0.87) & \\
\hline & & & $431.64(3.79)$ & $503.95(10.61)$ & $801.40(523.22)$ & \\
\hline \multirow[t]{18}{*}{$\mathrm{Si}_{10}$} & \multirow[t]{6}{*}{$10 a$} & $C_{3 v}\left({ }^{1} A_{1}\right)$ & & & & \multirow{6}{*}{0.46} \\
\hline & & & $110.51(1.90)$ & $189.31(0.16)$ & $228.24(1.35)$ & \\
\hline & & & 243.64(0.14) & $270.09(0.27)$ & $305.10(1.49)$ & \\
\hline & & & $316.02(1.06)$ & $318.51(0.18)$ & $341.12(2.00)$ & \\
\hline & & & $351.17(4.99)$ & $387.62(0.42)$ & $426.80(0.20)$ & \\
\hline & & & $459.25(0.02)$ & $510.07(0.32)$ & & \\
\hline & $10 b$ & $T_{d}\left({ }^{1} A_{1}\right)$ & & & & \\
\hline & & & $98.10(8.03)$ & $203.50(1.91)$ & $299.79(3.46)$ & 0.42 \\
\hline & & & $546.14(22.05)$ & & & \\
\hline & $10 c$ & $C_{2}\left({ }^{1} A\right)$ & & & & \\
\hline & & & $101.52(0.01)$ & $103.27(0.15)$ & $116.33(1.78)$ & 0.45 \\
\hline & & & $133.66(0.02)$ & $210.44(1.42)$ & $212.20(0.87)$ & \\
\hline & & & $221.85(0.42)$ & $229.57(3.29)$ & 259.72(0.45) & \\
\hline & & & $264.72(0.26)$ & $286.64(1.55)$ & $295.28(2.08)$ & \\
\hline & & & $300.78(0.40)$ & $309.33(0.19)$ & $325.89(0.12)$ & \\
\hline & & & $344.38(4.21)$ & $348.26(1.31)$ & $373.22(0.67)$ & \\
\hline & & & $383.12(0.39)$ & $395.78(0.50)$ & $440.76(3.84)$ & \\
\hline & & & $475.49(0.61)$ & $524.86(1.32)$ & $533.19(1.12)$ & \\
\hline $\mathrm{Si}_{11}$ & $11 a$ & $C_{s}\left({ }^{1} A^{\prime}\right)$ & & & & \\
\hline & & & $69.40(0.26)$ & $97.15(6.49)$ & $119.32(0.17)$ & 0.48 \\
\hline & & & $124.63(0.54)$ & $140.27(0.83)$ & $177.48(0.11)$ & \\
\hline & & & $215.03(1.35)$ & $229.82(0.07)$ & $240.20(7.61)$ & \\
\hline & & & $244.78(1.82)$ & $259.02(0.59)$ & $261.36(0.15)$ & \\
\hline & & & $280.38(2.71)$ & $289.11(0.59)$ & $295.83(1.67)$ & \\
\hline & & & $316.73(4.63)$ & $325.23(1.15)$ & $328.26(0.10)$ & \\
\hline & & & $358.32(0.85)$ & $367.64(0.28)$ & $378.00(1.25)$ & \\
\hline & & & $392.65(0.002)$ & $412.75(10.72)$ & $429.90(5.09)$ & \\
\hline & & & $432.35(1.94)$ & $470.02(4.49)$ & $517.23(9.70)$ & \\
\hline & $11 b$ & $C_{2 v}\left({ }^{1} A_{1}\right)$ & & & & \\
\hline & & & $104.53(1.17)$ & $134.20(2.19)$ & $136.35(3.31)$ & 0.48 \\
\hline & & & $153.96(4.23)$ & $164.43(0.73)$ & $177.84(0.87)$ & \\
\hline & & & $222.28(0.78)$ & $229.28(0.15)$ & $259.98(1.50)$ & \\
\hline & & & $270.32(2.25)$ & $291.92(0.48)$ & $304.56(7.21)$ & \\
\hline & & & $320.46(0.05)$ & $335.13(0.01)$ & $336.64(0.82)$ & \\
\hline & & & $344.59(8.56)$ & $374.74(0.03)$ & $388.99(0.39)$ & \\
\hline & & & $432.26(0.37)$ & $432.74(0.01)$ & $472.00(6.56)$ & \\
\hline & & & $496.16(18.95)$ & $519.60(22.37)$ & & \\
\hline & $11 c$ & $C_{s}\left({ }^{1} A^{\prime}\right)$ & & & & \\
\hline & & & $51.32(2.61)$ & $69.05(0.61)$ & $106.88(0.17)$ & 0.47 \\
\hline & & & $114.82(2.59)$ & $129.32(2.43)$ & $163.01(0.50)$ & \\
\hline & & & $164.29(0.54)$ & $201.17(0.87)$ & $225.80(0.06)$ & \\
\hline & & & $228.96(0.69)$ & $238.83(1.73)$ & $241.71(1.10)$ & \\
\hline & & & $262.43(2.53)$ & $285.86(0.15)$ & $287.24(0.23)$ & \\
\hline & & & $304.84(0.74)$ & $341.84(0.03)$ & $364.25(0.09)$ & \\
\hline & & & $368.26(5.43)$ & $386.66(2.20)$ & $390.20(0.002)$ & \\
\hline & & & $401.69(11.69)$ & $427.14(0.29)$ & $431.83(6.47)$ & \\
\hline & & & $446.87(2.88)$ & $483.25(2.27)$ & $504.03(12.67)$ & \\
\hline & $11 d$ & $C_{2}\left({ }^{1} A\right)$ & & & & \\
\hline & & & $68.90(0.82)$ & $88.65(0.47)$ & $109.24(0.53)$ & 0.47 \\
\hline & & & $127.18(2.26)$ & $139.19(2.17)$ & $194.75(0.64)$ & \\
\hline
\end{tabular}


TABLE II. (Continued.)

\begin{tabular}{ccccc}
\hline \hline \multirow{2}{*}{ Cluster } & Isomer & PG (state) & Vibrational frequency (IR intensities) & ZPE (eV) \\
\hline & & $219.95(0.05)$ & $228.05(0.05)$ & $252.04(0.91)$ \\
& $252.39(1.46)$ & $260.18(0.85)$ & $262.62(4.36)$ & $305.01(1.14)$ \\
& $279.70(1.57)$ & $284.32(2.10)$ & $329.07(1.33)$ & $368.54(0.27)$ \\
& $310.71(0.12)$ & $314.60(0.32)$ & $348.85(0.08)$ & $483.36(9.54)$ \\
\hline
\end{tabular}

capped trigonal prism (TTP). Previously, Lee, Chang, and $\mathrm{Lee}^{35}$ and also Luo, Zhao, and Wang ${ }^{43}$ reported that the isomer $9 b\left(C_{2 v},{ }^{1} A_{1}\right)$ was a possible global minimum based on semiempirical methods.

At the MP2/6-31G(d) level, the vibrational frequency analysis indicates that the distorted TTP $\left(C_{2 v},{ }^{1} A_{1}\right)$ (Ref. $22)$ is unstable. However, it has been shown that the distorted TTP $\left(C_{2 v}\right)$ isomer, although unstable at the MP2/6-31G $(d)$ level, can be a favorable structural motif in some larger silicon clusters. ${ }^{22}$ The distorted tricapped octahedron $\left(C_{s},{ }^{1} A^{\prime}\right)$ (Refs. 27, 28, and 31) is also found unstable at the MP2/6-31G $(d)$ level as it transforms into $9 c\left(C_{s},{ }^{1} A^{\prime}\right)$, another form of distorted tricapped trigonal prism (TTP), in a full geometry optimization. To our knowledge, the distorted TTP $\left(C_{s},{ }^{1} A^{\prime}\right)$ isomer has not been reported in the literatures. The tricapped octahedron in triplet state,
$9 d\left(C_{3 v},{ }^{3} A_{2}\right)$, is a relatively high-energy local minimum, as predicted by Raghavachari and Kohlfing. ${ }^{27}$

\section{4. $N=10$}

$\mathrm{Si}_{10}$ is a magic-number cluster which has been extensively studied theoretically. ${ }^{27,28,30,31,35,36,38-40,43,45}$ Our calculation confirms that the isomer $10 a\left(C_{3 v},{ }^{1} A_{1}\right)$, a tetracapped trigonal prism, is the global minimum, as already predicted by many other groups. ${ }^{28,31,36,38,40}$ The isomer $10 b\left(T_{d},{ }^{1} A_{1}\right)$, a tetracapped octahedron, is a low-energy local minimum as shown by Raghavachari and Kohlfing. ${ }^{27}$ The isomer $10 c\left(C_{2},{ }^{1} A\right)$, obtained via a full geometry optimization from the geometric isomer $10 k\left(C_{s}\right)$ in Ref. 40, is a new low-energy isomer that not has been reported in the literatures. Its energy is close to that of the isomer $10 \mathrm{~b}$.

TABLE III. The energy separations for low-energy isomers of $\mathrm{Si}_{7}-\mathrm{Si}_{11}$.

\begin{tabular}{|c|c|c|c|c|c|c|}
\hline Cluster & Isomer & PG (state) & $\begin{array}{c}\mathrm{MP} 2 / 6-31 \mathrm{G}(d) \\
(\mathrm{eV})\end{array}$ & $\begin{array}{c}\operatorname{CCSD}(\mathrm{T}) / 6-31 \mathrm{G}(d) \\
(\mathrm{eV})\end{array}$ & $\begin{array}{c}\mathrm{MP} 2 / 6-311(2 d) \\
(\mathrm{eV})\end{array}$ & $\begin{array}{c}\operatorname{CCSD}(\mathrm{T}) / 6-311(2 d) \\
(\mathrm{eV})\end{array}$ \\
\hline \multicolumn{7}{|l|}{$\mathrm{Si}_{7}$} \\
\hline & $7 a$ & $D_{5 h}\left({ }^{1} A_{1}^{\prime}\right)$ & 0.00 & 0.00 & & \\
\hline & $7 b$ & $C_{3 v}\left({ }^{1} A_{1}\right)$ & 0.93 & 0.67 & & \\
\hline & $7 c$ & $C_{3 v}\left({ }^{1} A_{1}\right)$ & 2.17 & 1.71 & & \\
\hline & $7 d$ & $C_{2 v}\left({ }^{1} A_{1}\right)$ & 2.63 & 2.21 & & \\
\hline \multicolumn{7}{|c|}{$-20 \times-1)$} \\
\hline & $8 a$ & $C_{2 h}\left({ }^{1} A_{g}\right)$ & 0.00 & 0.00 & & \\
\hline & $8 b$ & $C_{2 v}\left({ }^{1} A_{1}\right)$ & 0.31 & 0.19 & & \\
\hline & $8 c$ & $C_{2}\left({ }^{1} A\right)$ & 0.58 & 0.52 & & \\
\hline & $8 d$ & $C_{2 v}\left({ }^{1} A_{1}\right)$ & 0.41 & 0.55 & & \\
\hline & $8 e$ & $D_{3 d}\left({ }^{3} A_{1 g}\right)$ & 0.68 & 0.58 & & \\
\hline & $8 f$ & $C_{3 v}\left({ }^{3} A_{2}\right)$ & 0.95 & 0.60 & & \\
\hline & $8 g$ & $C_{s}\left({ }^{1} A^{\prime}\right)$ & 0.52 & 0.62 & & \\
\hline & $8 h$ & $D_{2 d}\left({ }^{1} A_{1}\right)$ & 1.00 & 1.73 & & \\
\hline \multicolumn{7}{|c|}{$\begin{array}{ll}2 d(\lambda 1) \\
\end{array}$} \\
\hline & $9 a$ & $C_{2 v}\left({ }^{1} A_{1}\right)$ & 0.00 & 0.00 & & \\
\hline & $9 b$ & $C_{2 v}\left({ }^{1} A_{1}\right)$ & 0.60 & 0.74 & & \\
\hline & $9 c$ & $C_{s}\left({ }^{1} A^{\prime}\right)$ & 1.28 & 1.24 & & \\
\hline & $9 d$ & $C_{3 v}\left({ }^{3} A_{2}\right)$ & 2.07 & 1.60 & & \\
\hline \multicolumn{7}{|l|}{$\mathrm{Si}_{10}$} \\
\hline & $10 a$ & $C_{3 v}\left({ }^{1} A_{1}\right)$ & 0.00 & 0.00 & & \\
\hline & $10 b$ & $T_{d}\left({ }^{1} A_{1}\right)$ & 1.99 & 0.75 & & \\
\hline & $10 c$ & $C_{2}\left({ }^{1} A\right)$ & 1.27 & 0.81 & & \\
\hline \multicolumn{7}{|l|}{$\mathrm{Si}_{11}$} \\
\hline & $11 a$ & $C_{s}\left({ }^{1} A^{\prime}\right)$ & 0.00 & 0.00 & $0.00\left(11 a^{\prime}\right)$ & $0.09\left(11 a^{\prime}\right)$ \\
\hline & $11 b$ & $C_{2 v}\left({ }^{1} A_{1}\right)$ & 0.53 & 0.02 & $0.40\left(11 b^{\prime}\right)$ & $0.00\left(11 b^{\prime}\right)$ \\
\hline & $11 c$ & $C_{s}\left({ }^{1} A^{\prime}\right)$ & 0.21 & 0.22 & & \\
\hline & $11 d$ & $C_{2}\left({ }^{1} A\right)$ & 0.87 & 0.53 & & \\
\hline & $11 e$ & $C_{s}\left({ }^{1} A^{\prime}\right)$ & & & 0.00 & 0.03 \\
\hline
\end{tabular}




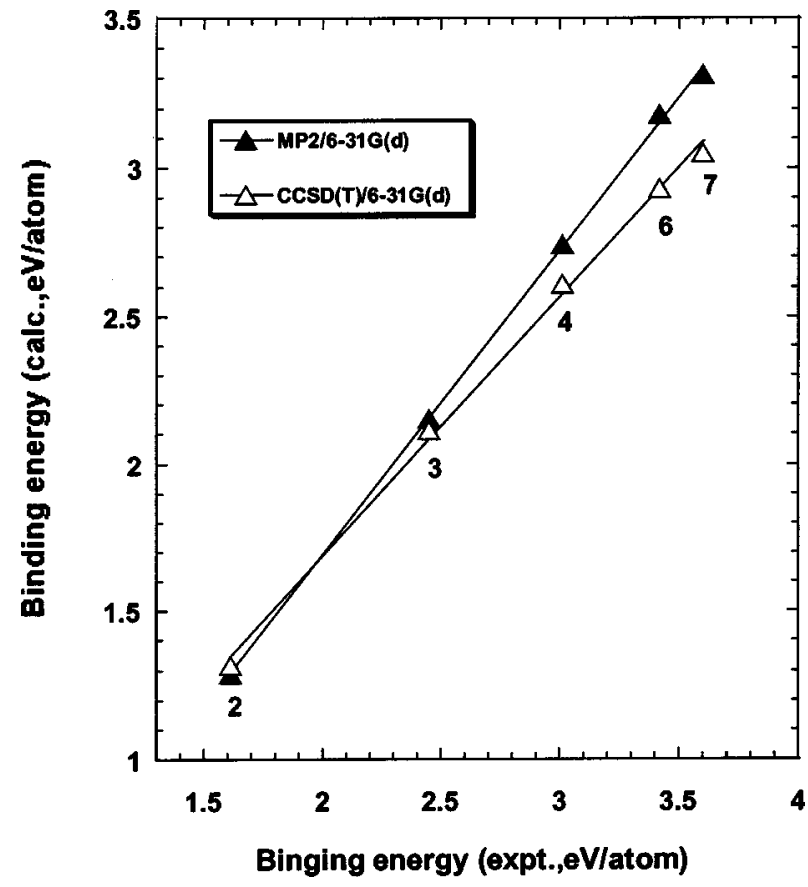

FIG. 2. Correlation of the calculated binding energies per atom and measured values for the low-energy isomers of $\mathrm{Si}_{n}(n=2-4,6-7)$.

\section{5. $N=11$}

$\mathrm{Si}_{11}$ deserves more discussion because the globalminimum structure of $\mathrm{Si}_{11}$ was not fully conclusive due to the existence of multiple nearly degenerate low-energy isomers. ${ }^{28,35,38,39,45}$ In fact, $\mathrm{Si}_{11}$ can be a good model system for testing theoretical methods. At the MP2/6-31G $(d)$ level we found that the $D_{3 h}$ pentacapped trigonal prism is unstable, although it is a stable structure when calculated at the HF/6-31G(d) level. ${ }^{28}$ Table III lists the total energy of three nearly isoenergetic geometric isomers calculated at the MP2/6-31G $(d) / / C C S D(T) / 6-31 G(d)$ level, $11 a\left(\mathrm{C}_{s},{ }^{1} A^{\prime}\right)$, a distorted tricapped tetragonal antiprism or a distorted pentacapped trigonal prism, $11 b\left(C_{2 v},{ }^{1} A_{1}\right)$, a tricapped trigonal prism with two additional caps on side trigonal faces, and $11 c\left(C_{s},{ }^{1} A^{\prime}\right)$, a bicapped tetragonal antiprism with an additional cap on one upper trigonal face. Among the three isomers, $11 a\left(C_{s},{ }^{1} A^{\prime}\right)$ was proposed by Lee, Chang, and $\mathrm{Lee}^{35}$ and also later by Sieck et al. $;^{45}$

TABLE IV. Binding energies per atom calculated at the MP2/6-31G $(d)$ and $\operatorname{CCSD}(\mathrm{T}) / 6-31 \mathrm{G}(d)$ levels compared with available experimental data.

\begin{tabular}{lcccc}
\hline \hline \multirow{2}{*}{$\begin{array}{l}\text { Cluster } \\
\text { (isomer) }\end{array}$} & Point group & $\mathrm{MP} 2 / 6-31 \mathrm{G}(d)$ & $\mathrm{CCSD}(\mathrm{T}) / 6-31 \mathrm{G}(d)$ & Expt. $^{\mathrm{a}}$ \\
\cline { 3 - 5 } & & 1.29 & 1.32 & 1.61 \\
$\mathrm{Si}_{2}$ & $D_{\infty h}$ & 2.15 & 2.11 & 2.45 \\
$\mathrm{Si}_{3}$ & $C_{2 v}$ & 2.74 & 2.61 & 3.01 \\
$\mathrm{Si}_{4}$ & $D_{2 h}$ & 3.18 & 2.93 & 3.42 \\
$\mathrm{Si}_{6}$ & $D_{4 h}$ & 3.31 & 3.05 & 3.60 \\
$\mathrm{Si}_{7}(7 a)$ & $D_{5 h}$ & 3.20 & 2.95 & \\
$\mathrm{Si}_{8}(8 a)$ & $C_{2 h}$ & 3.33 & 3.04 & \\
$\mathrm{Si}_{9}(9 a)$ & $C_{2 v}$ & 3.50 & 3.14 & \\
$\mathrm{Si}_{10}(10 a)$ & $C_{3 v}$ & 3.40 & 3.08 & \\
$\mathrm{Si}_{11}(11 a)$ & $C_{s}$ & & & \\
\hline \hline
\end{tabular}

${ }^{a}$ Reference 31 .
$11 b\left(C_{2 v},{ }^{1} A_{1}\right)$ and $11 c\left(C_{s},{ }^{1} A^{\prime}\right)$ were predicted by Rohlfing and Raghavachari ${ }^{28}$ based on the HF/6-31G $(d) / /$ MP4SDQ/6-31G $(d)$ calculation. The isomer $11 b\left(C_{2 v},{ }^{1} A_{1}\right)$ was also predicted to be a possible global minimum by Ho and coworkers ${ }^{39}$ using a density-functional pseudopotential theory within both local density and generalized gradient approximations; $11 a\left(C_{s},{ }^{1} A^{\prime}\right)$ [Cs(II) in Ref. 39] was predicted to be a local minimum. Ho and co-workers ${ }^{39}$ recently used Car-Parrinello molecular dynamics combined with the simulated annealing method to search for the global-minimum structure of $\mathrm{Si}_{11}$. They found again that $11 b$ is most likely the global minimum. At the MP2/6-31G(d)//CCSD(T)/6-31G $(d)$ level, we found that both isomers $11 a$ and $11 b$ are the possible global minimum while $11 c$ is only about $0.2 \mathrm{eV}$ higher in energy than $11 a$ and $11 b$. The isomer $11 d\left(C_{2},{ }^{1} A\right)$, another capped trigonal prism, is a new low-energy isomer whose energy is $0.53 \mathrm{eV}$ higher than that of $11 a$.

The fact that $\mathrm{Si}_{11}$ has at least three possible candidates for the global-minimum structure on the basis of MP2/6-31G $(d) / / C C S D(T) / 6-31 G(d)$ calculation prompts us to further examine the relative stability of these three geometric isomers with a larger basis set. We chose MP2/6-311G(2d)//CCSD(T)/6-311G(2d) levels for which our current computer facility can afford. The full geometry optimization at the MP2/6-311G(2d) level reveals that the previously optimized structure of $11 a$ and $11 b$ remains nearly unchanged [the newly optimized structures, called $11 a^{\prime}\left(C_{s},{ }^{1} A_{1}\right)$ and $11 b^{\prime}\left(C_{2 v},{ }^{1} A_{1}\right)$, are given in Table I(b)]. However, significant structural change occurred for the isomer $11 c\left(C_{s},{ }^{1} A^{\prime}\right)$. The newly optimized structure starting from $11 c$ is hereafter called $11 e\left(C_{s},{ }^{1} A^{\prime}\right)$, which is also a capped trigonal prism. The structural data of $11 e$ is shown in Table I(b). The calculated single-point energies for $11 a^{\prime}$, $11 b^{\prime}$, and $11 e$ at both the MP2/6-311G(2d) and $\operatorname{CCSD}(\mathrm{T}) / 6-311 \mathrm{G}(2 d)$ levels are listed in Table III. At the MP2/6-311G $(2 d)$ level, now both $11 a^{\prime}\left(C_{s},{ }^{1} A^{\prime}\right)$ and $11 e\left(C_{s},{ }^{1} A^{\prime}\right)$ are the two lowest-energy structures, whereas at the $\operatorname{CCSD}(\mathrm{T}) / 6-311 \mathrm{G}(2 d)$ level $11 b^{\prime}\left(C_{2 v},{ }^{1} A_{1}\right)$ becomes the global minimum but $11 e\left(C_{s},{ }^{1} A^{\prime}\right)$ is merely $0.03 \mathrm{eV}$ higher in energy than $11 b^{\prime}$. It can be also seen from Table III that the use of the larger basis set at the MP2 level $[6-311 \mathrm{G}(2 d)]$ results in a smaller energy difference between $11 b^{\prime}$ and $11 a^{\prime}$, compared to that between $11 b$ and $11 a$ at the MP2/6-31G(d) level, while at the $\operatorname{CCSD}(\mathrm{T}) / 6-311 \mathrm{G}(2 d)$ level $11 b^{\prime}$ becomes the global minimum. From this trend, it appears that the isomer $11 b^{\prime}$ would be more energetically favorable if an even larger basis set [e.g., 6-311(3df )] were used. Note that the difference in the zero-point vibrational energy for the three geometric isomers is less than $0.01 \mathrm{eV}$ (see Table II). Therefore, including zeropoint vibrational energy in the total energy will not qualitatively affect the energy ordering among the three nearly isoenergetic isomers. Note also that the structure of newly obtained $11 e\left(C_{s},{ }^{1} A^{\prime}\right)$ does not resemble that of $\mathrm{Cs}(\mathrm{I})$ isomer reported in Ref. 39, which was predicted to be another possible global minimum of $\mathrm{Si}_{11}$ [the $\mathrm{Cs}(\mathrm{I})$ 's geometric structure resembles that of $\left.C_{2 v} 11 b\right] .^{59}$

To summarize, our calculations at both the MP2 and 
$\operatorname{CCSD}(\mathrm{T})$ levels consistently reproduce all the globalminimum structures of $\mathrm{Si}_{7}-\mathrm{Si}_{10}$ reported in the literature. ${ }^{27,39,45}$ For $\mathrm{Si}_{11}$, however, the predicted globalminimum structure is different on the basis of the MP2 level and the $\operatorname{CCSD}(\mathrm{T})$ level. The $\operatorname{CCSD}(\mathrm{T}) / 6-311 \mathrm{G}(2 d)$ calculation indicates that the isomer $11 b^{\prime}\left(C_{2 v},{ }^{1} A_{1}\right)$ is the global minimum, but also shows that $11 e\left(C_{s},{ }^{1} A^{\prime}\right)$ is a very competitive candidate for the global minimum.

\section{B. Cluster growth feature}

As shown in Fig. 1, the pentagonal bipyrimid, tetrahedron, distorted octahedron, and tricapped trigonal prism (TTP) all could be viewed as motifs for low-energy structures of $\mathrm{Si}_{7}-\mathrm{Si}_{11}$. For example, the isomer $7 a\left(D_{5 h},{ }^{1} A_{1}^{\prime}\right)$ appears to be such a motif due to its high stability. Lowenergy clusters such as $8 d, 8 g, 9 a$, and $9 b$ can be constructed via capping $7 a$ on edges or faces. The distorted octahedron is the global minimum of $\mathrm{Si}_{6} \cdot{ }^{20,25}$ When capped on edges or faces, it becomes clusters $7 c, 7 d, 8 e, 8 h, 9 d$, or $10 \mathrm{~b}$. Although the tetrahedron $\left(\mathrm{Si}_{4}\right)$ is not a stable structure itself due to large strain energy, ${ }^{27}$ it can also be viewed as a building block for some low-energy structures (e.g., $7 b$ and $8 f$ ) when its faces are properly capped. Moreover, the TTP in triplet state is a local minimum of $\mathrm{Si}_{9}$, but it has a higher energy compared to many other low-energy isomers. Nevertheless, it can be viewed as a motif for some lowenergy structures of $\mathrm{Si}_{11}(11 a-11 e)$ and even some larger $\mathrm{Si}_{n}(n=12-26)$ clusters $^{21}$ as well.

A common feature in small silicon clusters $\mathrm{Si}_{7}-\mathrm{Si}_{11}$ is that the lowest-energy structures are all spherical-like. The spherical-like shape minimizes the surface area and reduces the number of dangling bonds. ${ }^{46}$ The low-energy structures of $\mathrm{Si}_{7}$, for example, deviate gradually from spherical-like shape, as the energy increases from isomer $7 a$ to $7 d$ (see Table III), indicating that the more spherical-like isomer $7 a$ is more energetically favorable. This deviation from spherical-like shape ( $7 a$ to $7 d$ ) is also manifested in their dipole moments (see Table V). As shown in Fig. 3, the binding energy per atom for the lowest-energy structure of $\mathrm{Si}_{n}$ $(n=2-11)$ increases with $n^{-1 / 3}$, supporting the sphericallike cluster growth. ${ }^{24}$ For nonspherical-like (prolate) cluster growth, the binding energy per atom would be nearly independent of the size of cluster.

In most cases, if clusters favor spherical-like shape the lowest-energy structures of the clusters tend to have high degree of symmetry. However, high symmetrical clusters with incomplete electronic shells can be even more stabilized through a geometry deformation which results in a lower degree of symmetry. ${ }^{24}$ As an example, the lowest-energy structure of $\mathrm{Si}_{8}$ does not exhibit cubic symmetry with the $O_{h}$ point group. Rather, it exhibits $C_{2 h}$ symmetry due to JahnTeller distortion, similar to the cases of $\mathrm{Si}_{4}$ (Ref. 27) and $\mathrm{Si}_{6}$ (Ref. 20). For $\mathrm{Si}_{9}$, the global minimum is $9 a$ with $C_{2 v}$ symmetry; it can be derived from the distortion of tricapped octahedron $\left(C_{3 v}\right)$ in singlet state.
TABLE V. Dipole moments of low-energy isomers of $\mathrm{Si}_{7}-\mathrm{Si}_{11}$ at the MP2/6-31G $(d)$ level.

\begin{tabular}{lccc}
\hline \hline Cluster & Isomer & PG $($ state $)$ & $\mu(\mathrm{D})$ \\
\hline $\mathrm{Si}_{7}$ & $7 a$ & $D_{5 h}\left({ }^{1} A_{1}^{\prime}\right)$ & 0.000 \\
& $7 b$ & $C_{3 v}\left({ }^{1} A_{1}\right)$ & 1.518 \\
& $7 c$ & $C_{3 v}\left({ }^{1} A_{1}\right)$ & 1.602 \\
$\mathrm{Si}_{8}$ & $7 d$ & $C_{2 v}\left({ }^{1} A_{1}\right)$ & 1.639 \\
& $8 a$ & $C_{2 h}\left({ }^{1} A_{g}\right)$ & 0.000 \\
& $8 b$ & $C_{2 v}\left({ }^{1} A_{1}\right)$ & 0.611 \\
& $8 c$ & $C_{2}\left({ }^{1} A\right)$ & 1.360 \\
& $8 d$ & $C_{2 v}\left({ }^{1} A_{1}\right)$ & 1.929 \\
& $8 e$ & $D_{3 d}\left({ }^{3} A_{1 g}\right)$ & 0.000 \\
& $8 f$ & $C_{3 v}\left({ }^{3} A_{2}\right)$ & 0.324 \\
$\mathrm{Si}_{9}$ & $8 g$ & $C_{s}\left({ }^{1} A^{\prime}\right)$ & 0.880 \\
& $8 h$ & $D_{2 d}\left({ }^{1} A_{1}\right)$ & 0.000 \\
& $9 a$ & $C_{2 v}\left({ }^{1} A_{1}\right)$ & 0.430 \\
$\mathrm{Si}_{10}$ & $9 b$ & $C_{2 v}\left({ }^{1} A_{1}\right)$ & 1.216 \\
& $9 c$ & $C_{s}\left({ }^{1} A^{\prime}\right)$ & 1.235 \\
& $9 d$ & $C_{3 v}\left({ }^{3} A_{2}\right)$ & 0.897 \\
$\mathrm{Si}_{11}$ & $10 a$ & $C_{3 v}\left({ }^{1} A_{1}\right)$ & 1.101 \\
& $10 b$ & $T_{d}\left({ }^{1} A_{1}\right)$ & 0.000 \\
& $10 c$ & $C_{2}\left({ }^{1} A\right)$ & 1.148 \\
& $11 a$ & $C_{s}\left({ }^{1} A^{\prime}\right)$ & 1.258 \\
& $11 b$ & $C_{2 v}\left({ }^{1} A_{1}\right)$ & 2.098 \\
\hline \hline
\end{tabular}

\section{Energy and stability}

It can be seen from Table III that the lowest-energy structures of $\mathrm{Si}_{7}-\mathrm{Si}_{11}$ all have closed-shell structures. It is known that the CCSD(T) level of theory ${ }^{52,56-58}$ is more accurate in estimating electron correlation than the MP2 level. In many cases, the $\operatorname{CCSD}(\mathrm{T})$ level yields only small quantitative changes in the relative energy of geometric isomers. For instance, isomers $7 b$ and $7 c$ of $\mathrm{Si}_{7}$ are higher in energy

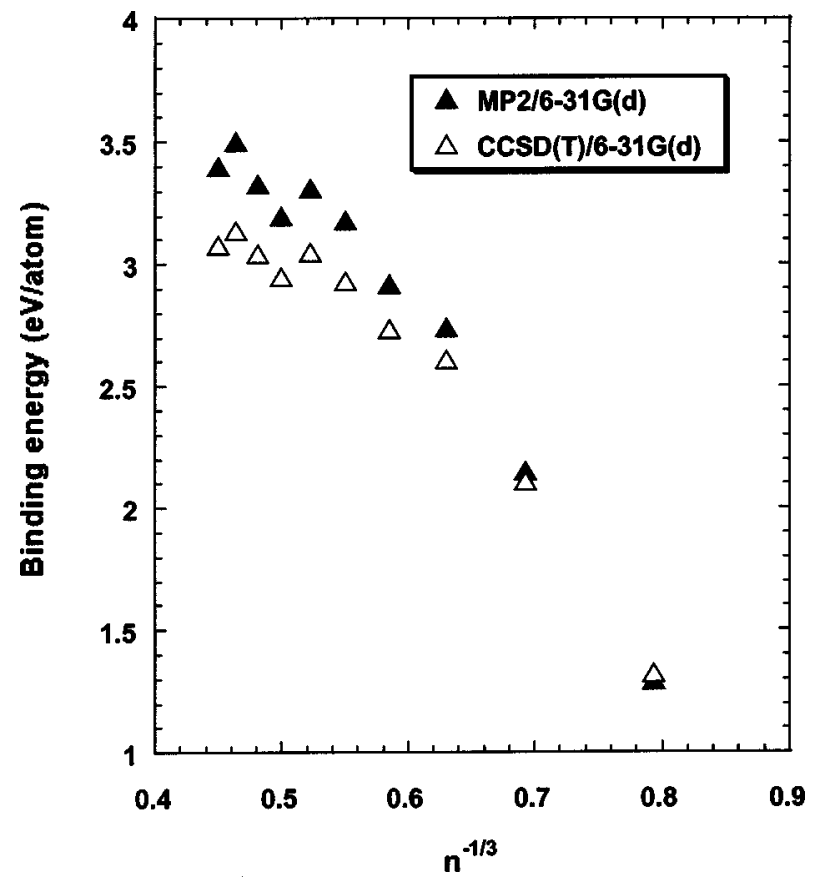

FIG. 3. Correlation of binding energy per atom and $n^{-1 / 3}$ for the low-energy isomers of $\mathrm{Si}_{2}-\mathrm{Si}_{11}$. 
than $7 a$ by 0.93 and $2.17 \mathrm{eV}$ at the MP2/6-31G $(d)$ level, but at the $\operatorname{CCSD}(\mathrm{T}) / 6-31 \mathrm{G}(d)$ level, $7 b$ and $7 c$ are 0.67 and $1.71 \mathrm{eV}$ higher than $7 a$. However, for some isomers of $\mathrm{Si}_{7}-\mathrm{Si}_{11}$ the CCSD(T) level can qualitatively affect the relative energy, that is, the MP2/6-31G $(d)$ energy ordering can be different from the $\operatorname{CCSD}(\mathrm{T}) / 6-31 \mathrm{G}(d)$ one. This shows the importance of having accurate electron-correlation energy in the determination of the relative stability for geometric isomers with very close energy, for example, the three nearly isoenergetic isomers of $\mathrm{Si}_{11}$.

The ionization potentials (IPs) can also be used to evaluate the relative stability of isomers. In principle, cluster IP should decrease gradually towards the work function of bulk phase as the size of clusters increase. In the case of metal clusters, ${ }^{47}$ the classical conducting spherical droplet (CSD) model suggests a linear IP versus $n^{-1 / 3}$ relationship, which can reproduce many experimental IPs quite well. However, the experimental IPs of $\mathrm{Si}_{n}(n=2-200)$ deviate notably from the CSD model. ${ }^{47} \mathrm{We}$ cannot offer a theoretical explanation for this deviation because of the limited size range of $\mathrm{Si}_{n}(n=7-11)$ considered here. But we have found an agreement between the calculated vertical ionization potentials (VIP) and measured values ${ }^{48}$ for $\mathrm{Si}_{2}-\mathrm{Si}_{11}$, as shown in Table VI. It is interesting to note that there is a large gap between $n=20$ and $n=22$ in the photoionization threshold measurements of $\mathrm{Si}_{n}(n=2-400),{ }^{49}$ which might imply a possible structural transition in this size range of silicon clusters. An ab initio molecular-orbital study for this size range of $\mathrm{Si}$ clusters is under way.

It is known that some simple-metal clusters are "magicnumber" clusters ${ }^{32}$ when the number of valence electrons in the clusters is $8,20,40$, or 58 etc. The magic-number clusters can be understood on the basis of particle-in-box model or jellium model. ${ }^{50}$ The "magic-number" behavior of small silicon clusters has been correlated with the trend of binding energy per atom as a function of cluster size. ${ }^{27}$ Luo, Zhao, and Wang ${ }^{43}$ pointed out that two factors can play major role in the cluster stability, one is the electronic configuration of an atom and another is the number of atoms in the cluster. Here, the binding energies of the lowest-energy structures of $\mathrm{Si}_{2}-\mathrm{Si}_{11}$ are calculated at the $\mathrm{MP} 2 / 6-31 \mathrm{G}(d)$ and $\operatorname{CCSD}(\mathrm{T}) / 6-31 \mathrm{G}(d)$ level, as shown in Table IV. Figure 4

TABLE VI. Vertical ionization potentials of the lowest-energy isomer of $\mathrm{Si}_{7}-\mathrm{Si}_{11}$.

\begin{tabular}{lllll}
\hline \hline Cluster & Isomer & PG (state) & $\begin{array}{c}\text { IP (calc. }) \\
(\mathrm{eV})\end{array}$ & $\begin{array}{c}\text { IP }(\text { expt. })^{\mathrm{a}} \\
(\mathrm{eV})\end{array}$ \\
\hline $\mathrm{Si}_{2}$ & & $D_{\infty h}$ & 7.87 & $>8.49$ \\
$\mathrm{Si}_{3}$ & & $C_{2 v}$ & 7.88 & $>8.49$ \\
$\mathrm{Si}_{4}$ & & $D_{2 h}$ & 8.09 & $7.97-8.49$ \\
$\mathrm{Si}_{5}$ & & $D_{3 h}$ & 8.02 & $7.97-8.49$ \\
$\mathrm{Si}_{6}$ & & $D_{4 h}$ & 7.84 & $7.97-8.49$ \\
$\mathrm{Si}_{7}$ & $7 a$ & $D_{5 h}\left({ }^{1} A_{1}^{\prime}\right)$ & 7.86 & $\sim 7.90$ \\
$\mathrm{Si}_{8}$ & $8 a$ & $C_{2 h}\left({ }^{1} A_{g}\right)$ & 7.16 & $7.46-7.87$ \\
$\mathrm{Si}_{9}$ & $9 a$ & $C_{2 v}\left({ }^{1} A_{1}\right)$ & 7.53 & $7.46-7.87$ \\
$\mathrm{Si}_{10}$ & $10 a$ & $C_{3 v}\left({ }^{1} A_{1}\right)$ & 7.95 & $\sim 7.90$ \\
$\mathrm{Si}_{11}$ & $11 a$ & $C_{s}\left({ }^{1} A^{\prime}\right)$ & 7.03 & $7.46-7.87$ \\
\hline \hline
\end{tabular}

${ }^{\mathrm{a}}$ Reference 50 .

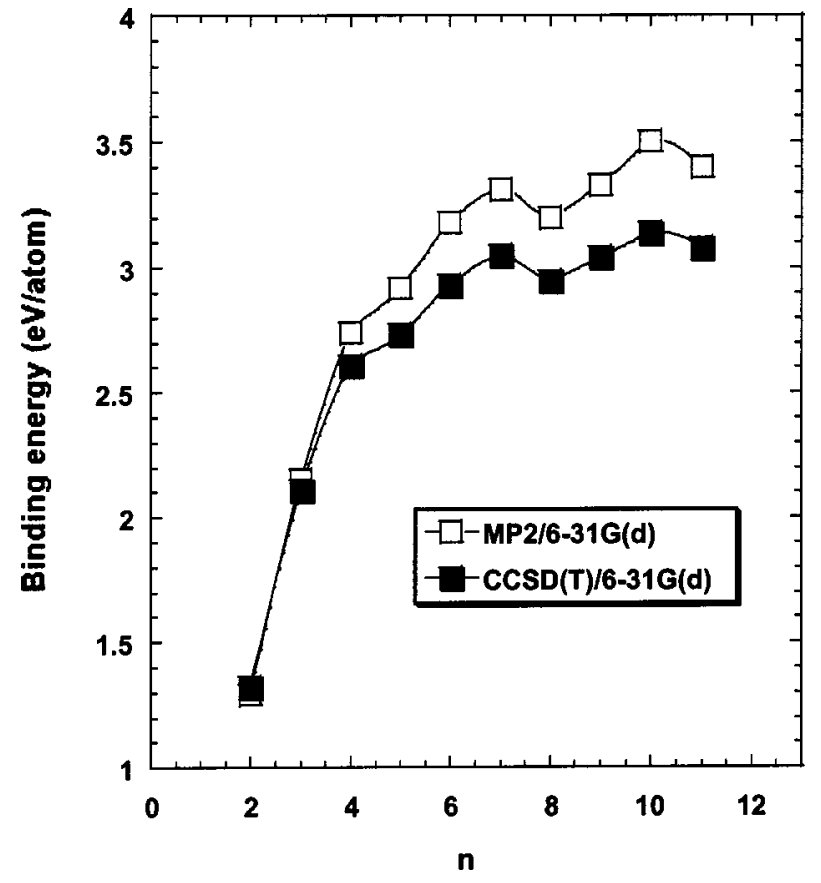

FIG. 4. Binding energy per atom of the lowest-energy structures of $\mathrm{Si}_{2}-\mathrm{Si}_{11}$ vs cluster size $n$.

plots the binding energies per atom versus the number of $\mathrm{Si}$ atoms $n$. A notable decrease in the slope of the curve can be seen at the cluster size of $\mathrm{Si}_{4}, \mathrm{Si}_{7}$, and $\mathrm{Si}_{10}$.

As pointed out by Raghavachari and Rohlfing, ${ }^{27}$ a better way to show the relative local stability of small silicon clusters is through the use of the incremental binding energy as a function of cluster size. The incremental binding energy can be defined as $\left(E_{n-1}+E_{1}\right)-E_{n}$, where $E_{n}$ is the single-point total energy of cluster $\mathrm{Si}_{n}$. Figure 5 shows the calculated incremental binding energy versus cluster size $n$ at both

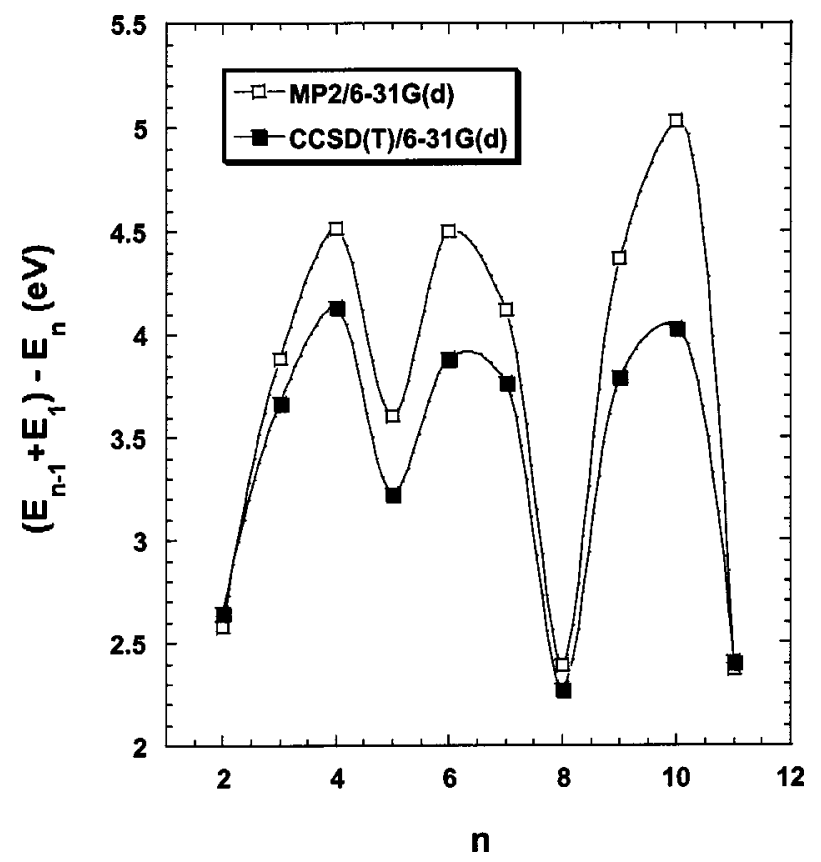

FIG. 5. Incremental binding energy of $\mathrm{Si}_{2}-\mathrm{Si}_{11}$ clusters vs cluster size $n$. 


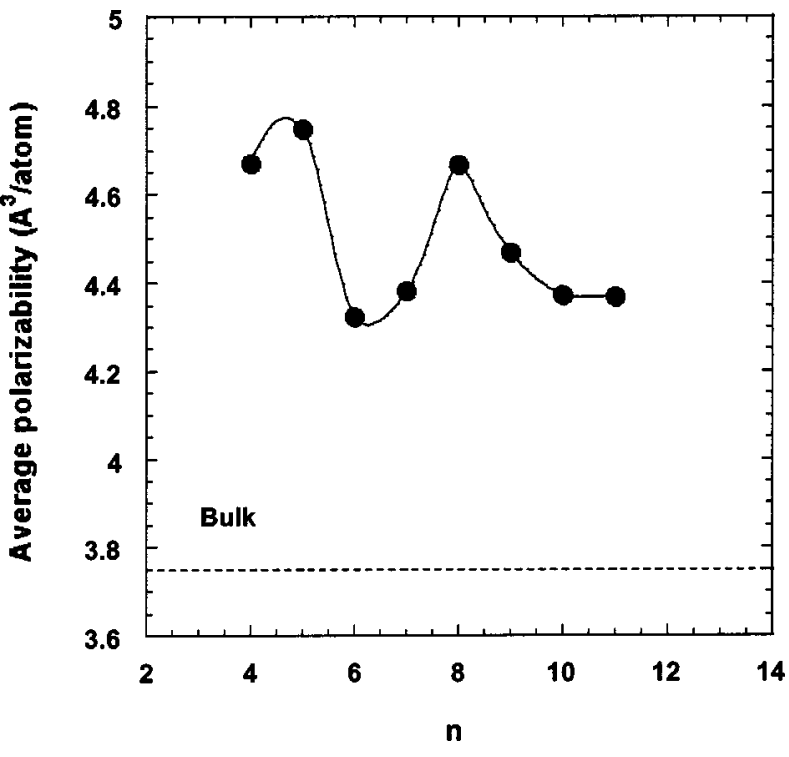

FIG. 6. Average polarizability for the lowest-energy structures of $\mathrm{Si}_{4}-\mathrm{Si}_{11}$ as a function of cluster size $n$.

MP2/6-31G $(d)$ and $\operatorname{CCSD}(\mathrm{T}) / 6-31 \mathrm{G}(d)$ levels. Clearly, the local maximum in the incremental binding energy corresponds to the cluster $\mathrm{Si}_{4}, \mathrm{Si}_{6}$, and $\mathrm{Si}_{10}$. For the latter, the marked local stability together with the fact that $\mathrm{Si}_{10}$ has the highest binding energy compared to other small silicon clusters provide reasonable explanation why $\mathrm{Si}_{10}$ is a magicnumber cluster with exceptional stability.

The cluster polarizability stems mainly from the surface electron density of the cluster. ${ }^{42}$ As mentioned above, smaller clusters have a larger surface/interior atom ratio. For $\mathrm{Si}_{7}-\mathrm{Si}_{11}$, nearly all atoms are on the surface of the clusters. Thus, the polarizability of these clusters is expected to be larger than that of the bulk. In addition, the unsaturated bonds at the cluster surface will make polarizabilities even larger. In Fig. 6, results are shown for the size dependence of mean polarizability for $\mathrm{Si}_{4}-\mathrm{Si}_{11}$. The relatively strong stability of the magic-number cluster $\mathrm{Si}_{6}$ and $\mathrm{Si}_{10}$ seems to correlate well with the local minimum in the cluster polarizability versus $n$.

\section{CONCLUSIONS}

We have obtained optimized geometries of a number of low-energy geometric isomers of $\mathrm{Si}_{7}-\mathrm{Si}_{11}$ at the MP2/6-31G $(d)$ level and their energy at the $\operatorname{CCSD}(\mathrm{T}) / 6-31 \mathrm{G}(d)$ level. For $\mathrm{Si}_{11}$, a larger basis set with both levels of theory, MP2/6-311G $(2 d)$ and CCSD $(\mathrm{T}) / 6-311 \mathrm{G}(2 d)$, were also employed. In most cases the electron-correlation effects on geometries of clusters are only quantitative, compared to geometries obtained at the $\mathrm{HF} / 6-31 \mathrm{G}(d)$ level. In some cases, the electron-correlation effects can be qualitative. This is particularly important for $\mathrm{Si}_{11}$. The calculated binding energies and vertical ionization potentials for the lowest-energy structures of $\mathrm{Si}_{7}-\mathrm{Si}_{11}$ are in agreement with experimental values. The binding energy per atom has an $n^{-1 / 3}$ dependence, suggesting that small silicon clusters favors spherical-like cluster growth. A few new lowenergy geometric isomers are found for these silicon clusters.
Among them, a new candidate for the global minimum of $\mathrm{Si}_{11}$ is identified. The relatively strong stability of magicnumber clusters $\mathrm{Si}_{n}(n=6$ and 10) appears to correlate well with their relatively higher binding energy per atom, their local-maximum feature in the incremental binding energy and their local-minimum feature in the cluster polarizability vs the cluster size $n$.

\section{ACKNOWLEDGMENTS}

The authors thank Professor K. Raghavachari, Professor B. Pan, and S. Yoo for valuable discussions. This work is supported by the National Science Foundation and by the research computing facility at the University of NebraskaLincoln.

${ }^{1}$ T. G. Dietz, M. A. Duncan, D. E. Powers, and R. E. Smally, J. Chem. Phys. 74, 6511 (1981)

${ }^{2}$ M. D. Morse, J. B. Hopkins, P. R. R. Langridge-Smith, and R. E. Smalley, J. Chem. Phys. 79, 5316 (1983)

${ }^{3}$ J. L. Gole, J. H. English, and V. E. Bondybey, J. Phys. Chem. 86, 2560 (1982)

${ }^{4}$ V. E. Bondybey and J. H. English, J. Chem. Phys. 80, 568 (1984).

${ }^{5}$ D. J. Trevor, R. L. Whetten, D. M. Cox, and A. Kaldor, J. Am. Chem. Soc. 107, 5181 (1985).

${ }^{6}$ S. J. Riley, E. K. Parks, G. C. Nieman, L. G. Pobo, and S. Wexler, J. Chem. Phys. 80, 1360 (1984).

${ }^{7}$ M. F. Jarrold, Science 252, 1085 (1991).

${ }^{8}$ W. L. Brown, R. R. Freeman, K. Raghavachari, and M. Schluter, Science 235, 860 (1987)

${ }^{9}$ S. Hayashi, Y. Kanzawa, M. Kataoka, T. Nagarede, and K. Yamamoto, Z. Phys. D: At., Mol. Clusters 26, 144 (1993).

${ }^{10}$ O. Cheshnovsky, S. H. Yang, C. L. Pettiette, M. J. Craycraft, Y. Liu, and R. E. Smalley, Chem. Phys. Lett. 138, 119 (1987).

${ }^{11}$ J. R. Heath, Y. Liu, S. C. O'Brien, Q. Zhang, R. F. Curl, F. K. Tittel, and R. E. Smalley, J. Chem. Phys. 83, 5520 (1985).

${ }^{12}$ Q. L. Zhang, Y. Liu, R. F. Curl, F. K. Tiittel, and R. E. Smalley, J. Chem. Phys. 88, 1670 (1988)

${ }^{13}$ L. A. Bloomfield, R. R. Freeman, and W. L. Brown, Phys. Rev. Lett. 54, 2246 (1985)

${ }^{14}$ L. A. Bloomfield, M. E. Guesic, R. R. Freeman, and W. L. Brown, Chem. Phys. Lett. 121, 33 (1985).

${ }^{15}$ K. D. Rinnen and M. L. Mandich, Phys. Rev. Lett. 69, 1823 (1992).

${ }^{16}$ W. Begemann, K. H. Meiwes-Broer, and H. O. Lutz, Phys. Rev. Lett. 73, 2248 (1986)

${ }^{17}$ M. F. Jarrold and E. C. Honea, J. Phys. Chem. 95, 9181 (1991).

${ }^{18}$ J. M. Hunter, J. L. Fye, M. F. Jarrold, and J. E. Bower, Phys. Rev. Lett. 73, 2063 (1994).

${ }^{19}$ T. P. Martin and H. Schaber, J. Chem. Phys. 83, 855 (1985).

${ }^{20}$ E. C. Honea, A. Ogura, C. A. Murray, K. Raghavachari, W. O. Sprenger, M. F. Jarrold, and W. L. Brown, Nature (London) 366, 42 (1993).

${ }^{21}$ S. Li, R. J. Van Zee, W. Weltner, and K. Raghavachari, Jr., Chem. Phys. Lett. 243, 275 (1995).

${ }^{22}$ K. M. Ho, A. A. Shvartsburg, B. Pan, Z. Y. Lu, C. Z. Wang, J. G. Wacker, J. L. Fye, and M. F. Jarrold, Nature (London) 392, 582 (1998).

${ }^{23}$ M. F. Jarrold and V. A. Constant, Phys. Rev. Lett. 67, 2994 (1991); M. F. Jarrold and J. E. Bower, J. Phys. Chem. 96, 9180 (1992); M. F. Jarrold, J. Phys. Chem. 99, 11 (1995).

${ }^{24} \mathrm{H}$. Haberland, Clusters of Atoms and Molecules: Theory, Experiment, and Clusters of Atoms (Springer Verlag, New York, 1994).

${ }^{25}$ K. Raghavachari and V. Logovinsky, Phys. Rev. Lett. 55, 2853 (1985).

${ }^{26}$ K. Raghavachari, J. Chem. Phys. 84, 5672 (1986).

${ }^{27}$ K. Raghavachari and C. M. Rohlfing, J. Chem. Phys. 89, 2219 (1988).

${ }^{28}$ C. M. Rohlfing and K. Raghavachari, Chem. Phys. Lett. 167, 559 (1990); J. Chem. Phys. 96, 2114 (1992).

${ }^{29}$ K. Raghavachari and C. M. Rohlfing, Chem. Phys. Lett. 94, 3670 (1991).

${ }^{30}$ K. Raghavachari and C. M. Rohlfing, Chem. Phys. Lett. 198, 521 (1992).

${ }^{31}$ J. C. Grossman and L. Mitas, Phys. Rev. Lett. 74, 1323 (1995); L. Mitas, J. C. Grossman, I. Stich, and J. Tobik, Phys. Rev. Lett. 84, 1479 (2000).

${ }^{32}$ W. D. Knight, K. Clemenger, W. A. de Heer, W. A. Saunders, M. Y. Chou, and M. L. Cohen, Phys. Rev. Lett. 52, 2141 (1984). 
${ }^{33}$ P. Ballone, W. Audreoni, R. Car, and M. Parrinello, Phys. Rev. Lett. 60, 271 (1988)

${ }^{34}$ U. Rothlisberger, W. Andreoni, and P. Giannozzi, J. Chem. Phys. 96, 1248 (1992).

${ }^{35}$ I. H. Lee, K. J. Chang, and Y. H. Lee, J. Phys.: Condens. Matter 6, 741 (1994).

${ }^{36}$ P. Ordejon, D. Lebedenko, and M. Menon, Phys. Rev. B 50, 5645 (1994).

${ }^{37}$ T. Fruuenheim, F. Weich, T. Kohler, S. Uhlmann, D. Porezag, and G. Seifert, Phys. Rev. B 52, 11492 (1995).

${ }^{38}$ D. J. Wales, Phys. Rev. A 49, 2195 (1994).

${ }^{39}$ B. Liu, Z. Y. Lu, B. Pan, C.-Z. Wang, K.-M. Ho, A. A. Shvartsburg, and M. F. Jarrold, J. Chem. Phys. 109, 9401 (1998); Z. Y. Lu, C. Z. Wang, and K. M. Ho, Phys. Rev. B 61, 2329 (2000).

${ }^{40}$ B. X. Li, P. L. Cao, and M. Jiang, Phys. Status Solidi B 218, 399 (2000); B. X. Li and P. L. Cao, Phys. Rev. A 62, 023201 (2000); B. X. Li and P. L. Cao, J. Phys.: Condens. Matter 13, 1 (2001).

${ }^{41}$ S. Wei, B. N. Barnett, and U. Landman, Phys. Rev. B 55, 7935 (1997).

${ }^{42}$ I. Vasiliev, S. Ogut, and J. R. Chelikowsky, Phys. Rev. Lett. 78, 4805 (1997).

${ }^{43}$ Y. Luo, J. Zhao, and G. H. Wang, Phys. Rev. B 60, 10703 (1999).

${ }^{44}$ G. Pacchioni and J. Koutecky, J. Chem. Phys. 84, 3301 (1986).

${ }^{45}$ A. Sieck, D. Porezag, Th. Frauenheim, M. R. Pederson, and K. Jackson, Phys. Rev. A 56, 4890 (1997).
${ }^{46}$ E. Kaxiras, Phys. Rev. Lett. 64, 551 (1990); E. Kaxiras and K. Jackson, Phys. Rev. Lett. 71, 727 (1993).

${ }^{47}$ C. Sebenne, D. Bolmont, G. Guichar, and M. Balkanski, Phys. Rev. B 12, 3280 (1975)

${ }^{48}$ M. M. Kappes, Chem. Rev. 88, 1271 (1988).

${ }^{49}$ K. Fuke, K. Tsukamoto, F. Misaizu, and M. Sanekata, J. Chem. Phys. 99, 7807 (1993).

${ }^{50}$ W. A. de Heer, W. D. Knight, M. Y. Chou, and M. L. Cohen, Solid State Phys. Vol. 40, edited by H. Ehrenreich, D. Turnbull (Academic, San Diego, 1987).

${ }^{51}$ M. Haser, J. AlmLof, and G. E. Scuseria, Chem. Phys. Lett. 181, 497 (1991).

${ }^{52}$ C. Moller and M. S. Plesset, Phys. Rev. 46, 618 (1934).

${ }^{53}$ W. J. Hehre, L. Radom, P. Von R. Schleyer and J. A. Pople, Ab Initio Molecular Orbital Theory (Wiley, New York, 1985).

${ }^{54}$ C. Peng, P. Y. Ayala, H. B. Schelgel, and M. J. Frisch, J. Comput. Chem. 17, 49 (1996).

${ }^{55}$ M. J. Frisch, G. W. Trucks, H. B. Schlegel et al., Gaussian 98, Revision A9, Gaussian, Inc., Pittsburgh, PA, 1998.

${ }^{56}$ J. A. Pople and M. Head-Gordon, J. Chem. Phys. 87, 5968 (1987).

${ }^{57}$ R. J. Bartlett, Annu. Rev. Phys. Chem. 32, 359 (1981).

${ }^{58}$ P. J. Knowles, C. Hampel, and H.-J. Werner, J. Chem. Phys. 99, 5219 (1993).

${ }^{59}$ B. Pan (private communication). 